\title{
Aeroelastic Control of Long-Span Suspension Bridges with Controllable Winglets
}

\author{
K.N. Bakis, M. Massaro, M.S. Williams, D.J.N. Limebeer
}

\begin{abstract}
The structural-aerodynamic modelling and dynamic stabilization of a three-dimensional suspension bridge model is considered. Our emphasis is on investigating the effectiveness of leading and trailing edge flaps in suppressing aeroelastic instabilities. The East Great Belt Bridge is chosen as a design example and its aeroelastic limits are computed using both thin aerofoil theory and flutter derivatives. The problem is cast in an efficient reduced size finite element formulation with aerodynamic forces expressed in the time domain by use of a high-fidelity rational function approximation. Circulatory aerodynamic forces are modeled using a feedback loop for every element and the problem is expressed in a form suitable for implementation of modern control techniques. The structure's full multimodal response is considered and numerical predictions show very good agreement against experimental data from the literature. In order to account for modelling errors and uncertainties while designing the controller, elements from robust control theory are invoked. The stability and robustness of the bridge when fitted with flaps controlled by optimal and suboptimal $H_{\infty}$ controllers is discussed for varying lengths of control surfaces along the suspended span as the optimum configuration for aerodynamic performance is investigated.
\end{abstract}

\section{Index Terms}

Long-Span Bridges, Thin Aerofoil Theory, Flutter, Torsional Divergence, Passive Aerodynamic Control, Erection Stage, Robust Control, Flaps, Humber Bridge

\section{INTRODUCTION}

It is well understood that aeroelastic instabilities like flutter and torsional divergence pose limits to the length increase of cable-supported bridges, with the now iconic Tacoma Narrows bridge disaster serving as a reminder of the importance of efficient aerodynamic design. Increasing span lengths pose a challenge to aeroelastic stability and experience gained from record span projects such as the Akashi Kaikyo Bridge [25] and Great Belt East Bridge showed that classic aerodynamic design either in the form of deep truss girders (Akashi Bridge) or the more modern flat box girder solution (Humber, Bosporus, Great Belt) reaches its limit for spans approaching 2000m [11]. To overcome these limitations appropriate countermeasures should be adopted, [26], which traditionally have focused on the deck's aerodynamic improvement or alternatively on the implementation of active and passive control methodologies.

Control applications to civil engineering structures have been gaining in popularity thanks to novel advances in the field [6] with a particular emphasis to bridge structures [8]. Control of flutter instability by active control surfaces was first proposed by Ostenfeld and Larsen in [30]. The premise is that rotational movement of control surfaces adjacent to the deck generate aerodynamic forces which aim at stabilizing deck movement. Further analysis was later undertaken by Wilde and Fujino $[42,43]$ where flaps were implemented on a deck segment. A Rational Function Approximation (RFA) was used to model the unsteadiness of the flow in the time-domain and a variable gain output feedback controller was implemented. Omenzetter et al. $[28,29]$ extended the flap configuration taking into account the

This work was partially funded by the IKY-State Scholarship Foundation by means of the ESPA European fund 2007-2013 and supported by a Marie Curie Intra European Fellowship within the 7th European Community Framework Programme. 
three-dimensionality of the bridge and a pure-gain passive mechanism was proposed. Passive puregain controllers are in principle easy to implement, but these systems forego the advantages that might accrue from phase compensation. Fixed-phase controllers, such as those derived in [15] are difficult to construct physically. Realizable systems that introduce frequency-dependent phase may operate satisfactorily, but this has not thus far been established using a full bridge model. more recently $\mathrm{Li}$ [22] performed an active control procedure using a pair of rotatable winglets at a distance from the deck. The feasibility of a passive control mechanism against flutter instability during erection of a suspension bridge has been investigated in [5] whereas some efforts have been made to combine the advantages of control surfaces with a Tuned Mass Damper (TMD) [20,37], although a detailed analysis has not been reported yet. Up to date there has been no practical application of implementing actively or passively controlled flaps to a bridge, because their reliability and effectiveness has not been clearly demonstrated.

In terms of the aerodynamic control of bridge structures three dimensional aeroelastic modelling by expressing unsteady aerodynamic forces in the frequency domain has been reported in $[4,27,36]$. The procedure involves algorithms such as the k-method and the p-k method inherited from the aeronautics industry, which are based in an iterative procedure for both the wind velocity and the frequency of the oscillating forces. A state-space approach that fits the unsteady part of the aerodynamic forces by use of rational function approximations [18] makes however the iterative process redundant. An implementation of this procedure to a full bridge structure has been presented by Omenzetter et al. [28] and Chen et al. [9] for thin aerofoil and flutter derivatives respectively.

This work extends previous analytic investigations [13,23] of a deck-flap system into a three dimensional bridge model having flaps of finite length along the main span. The emphasis in this paper is placed on active control schemes, which enables the determination of the minimum surfaces needed for aeroelastic control. In our work we build a high-fidelity rational approximation of the classical Theodorsen Circulation function which is used for every element of the bridge structure. The structural part of this work is based on Abdel Ghaffar's FE formulation [1-3] which is appropriately modified to account for a streamlined box girder (while the original formulation dealt with a truss girder). Forces on cables are computed by solving the nonlinear cable equation [16]. We validate the model by simulating the modal characteristics of the Great Belt Bridge and comparing them to previous studies. We later formulate the aeroelastic FE model by employing thin aerofoil theory [39] as well as expressing the aerodynamic loading through experimentally derived flutter derivatives [17]. For the first case, a high-fidelity quartic rational approximation of Theodorsen's circulation function is considered, and for the second case Roger's [31] approximation is used. Results show that the thin aerofoil assumption is very effective in predicting flutter instability limits for a flat box girder like the one herein considered. The afore-mentioned analyses also expose a non-oscillatory instability referred to as torsional divergence. The torsional divergence mode is an aerodynamic mode that goes unstable when there is a loss of torsional rigidity due to cancellation of the (positive) torsional stiffness of the structure by the negative pitch-related aerodynamic moment [7].

This paper places emphasis on formulating the full multimodal aeroelastic response by means of a reduced size model. A very good agreement in terms of modal responses is obtained with only 350 structural degrees of freedom. By using feedback for the unsteady circulatory component of the aerodynamics we reduce the number of aerodynamic states to half. For the case of the Great Belt (GB) cross section thin aerofoil theory and flutter derivatives give very close predictions to flutter onset thus corroborating the justification of the wing-aileron-tab transformation for modelling the flap aerodynamic contribution. Previous work including bridge stabilization with controllable surfaces either use passive systems, which have the advantage of not requiring power source, but use a simplistic control approach neglecting phase compensation and robustness properties or make use of 


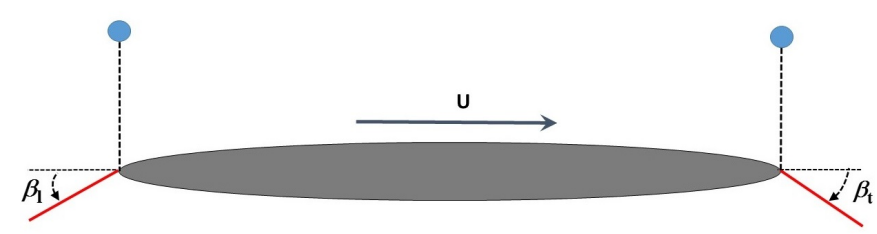

Fig. 1: Cross section of a streamlined long-span suspension bridge with flutter suppression controllable flaps. The wind speed is denoted as $U$, while the leading- and trailing-edge flap angle are denoted as $\beta_{l}$ and $\beta_{t}$, respectively.

active control techniques, optimal control, pole placement algorithm, which again neglect robustness issues. This work aims to optimize the closed-loop system's robustness to uncertainties, which can be interpreted as uncertainty in modal characteristics or aerodynamic parameters. Our goal is to establish an active control strategy that results in a minimum area of control surfaces along the span while attaining good robustness characteristics, Fig. 1. In conclusion it is shown that for wind speeds above the divergence speed there is a clear loss in uncertainty tolerance and in conclusion we demonstrate the effectiveness of the designed controllers by simulating the response in the time domain. The analysis presented in this paper is of analytical nature and concentrates on the control design process on a full FE bridge model. Experimental work is carried through in conjunction to this work, [12], to validate the numerical assumptions.

\section{Structural Model}

This section provides a procedure overview for constructing a full bridge model according to Abdel Ghaffar's structural framework [1-3] and compares the results to previous analyses of the Great Belt Bridge. The FE framework adopted provides a significant reduction of the computational burden because it accounts for the effect of the main cables and hangers for every deck element indirectly. The motion of long-span bridges in the vertical, lateral and torsional direction can with a high degree of accuracy be considered independent from one another so each case was treated separately and no coupling between them was assumed. In the pure vertical modes, all points of a given cross section move the same amount and remain in phase. In the torsional case the bridge section rotates about its centre point and for the lateral motion, each cross section swings in a pendular fashion in its own vertical plane and therefore there is an incidental upward movement of the cables and of the suspended structure.

\section{A. Modelling Methodology}

Fig.2a presents the degrees of freedom, vertical translation and rotation, corresponding to the vertical vibration case. Since the displacements of each stiffening structure node must equal the displacements of the corresponding cable node, it is appropriate to define only the nodes on the centre line of the deck element. Similar element configurations are defined for the lateral and torsional vibration cases. The general procedure for deriving the stiffness and mass matrices for a typical deck element consists firstly in expressing the potential and kinetic energy of the suspended structure and the cables separately for each vibration case. In order to formulate the FE approach the Hermitian cubic polynomials are chosen to build the shape functions of the element, expressing the vertical $v$, lateral $l$ and torsional $t$ displacements at every internal point as a function of the nodal displacements $q_{1 . .12}$ :

$$
\left\{\begin{array}{l}
v(x) \\
l(x) \\
t(x)
\end{array}\right\}=\left[\begin{array}{cccccccccccc}
H_{1} & H_{2} & 0 & 0 & 0 & 0 & H_{3} & H_{4} & 0 & 0 & 0 & 0 \\
0 & 0 & H_{1} & H_{2} & 0 & 0 & 0 & 0 & H_{3} & H_{4} & 0 & 0 \\
0 & 0 & 0 & 0 & 0 & H_{5} & 0 & 0 & 0 & 0 & 0 & H_{6}
\end{array}\right]\left\{\begin{array}{c}
q_{1} \\
\vdots \\
q_{12}
\end{array}\right\}
$$




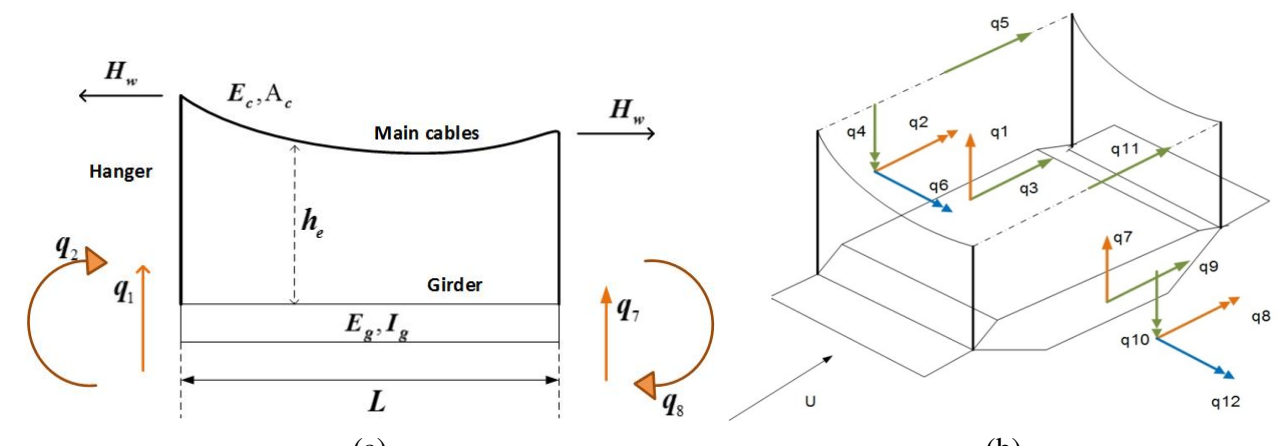

(a)

(b)

Fig. 2: (a) Nodal degrees of freedom of the FE element for the vertical vibration case. (b) All degrees of freedom of the FE element (orange $q_{1}, q_{2}, q_{7}, q_{8}$ correspond to the vertical, green $q_{3}, q_{4}, q_{5}, q_{9}, q_{10}, q_{11}$ to the lateral and blue $q_{6}, q_{12}$ to the torsional degrees of freedom).

where

$$
\begin{array}{cc}
H_{1}(x)=1-3 \frac{x^{2}}{L^{2}}+2 \frac{x^{3}}{L^{3}} & H_{2}(x)=-\left(x-2 \frac{x^{2}}{L}+\frac{x^{3}}{L^{2}}\right) \\
H_{3}(x)=3 \frac{x^{2}}{L^{2}}-2 \frac{x^{3}}{L^{3}} & H_{4}(x)=-\left(-\frac{x^{2}}{L}+\frac{x^{3}}{L^{2}}\right) \\
H_{5}(x)=1-\frac{x}{L} & H_{6}(x)=\frac{x}{L}
\end{array}
$$

with $0<x<L$ the position along the element spanwise length. The combination of all three vibration components (vertical $v$, lateral $l$, torsional $t$ ) culminates in an element having six degrees of freedom per node as illustrated in Fig.2b.

Implementing Eq.(1) in the potential and kinetic energy expressions yields the elemental mass matrices and elemental stiffness matrices expressing the elastic stiffness of the girder and the gravity stiffness of the cables, as well as a distributed matrix expressing the elastic stiffness of the cables. The gravity stiffness matrix is related to the energy stored in the cable due to the constant horizontal force. The elastic stiffness on the other hand stems from the additional cable tension caused by vibrations.

\section{B. Description of the Great Belt Bridge}

One key structural characteristic of the East Great Belt Bridge (1998, DK) is the continuous girder deck over the full span of $2694 \mathrm{~m}$ [21]. Suspension bridges of this type are usually hinged at the pylons in order to accommodate an expansion joint there. The Great Belt similarly to the Humber, Severn and Bosporus Bridge has a streamlined box girder deck section, which is expected to display to an aerofoil heave-pitch flutter mode. The bridge has a cable sag ratio 1:9, chosen to reduce sliding forces in the anchorages. It is also interesting to note that for the two approach bridges 32 TMDs were installed for suppressing vortex shedding each having a mass of $8 \mathrm{t}$ (approximately $0.5 \%$ of the modal mass). The structural parameters implemented in the FE model are presented in Tab.I.

\section{Modal Analysis Results for the Great Belt Bridge}

Fig.3 and Fig.4 compare the natural frequencies and shapes of the vertical, lateral and torsional modes obtained by implementing the FE model developed with the modes shapes and natural frequencies reported in Larsen [21]. Further modal analyses of the Great Belt are given in [19] which are the product of more detailed FE modelling using commercial packages. The proposed mathematical formulation however results in a model of considerably lower size while staying within the uncertainty scatter of more complex models. An equal displacement on the main cables is expected because the model assumes inextensibility of the hangers. It might be worth noting that the antisymmetric deflections of the cable and the stiffening structures cause no additional cable tension $H_{w}$ because 
TABLE I: Structural properties of the FE model of the Great Belt Bridge.

\begin{tabular}{lll}
\hline Element & Property & Value \\
\hline \multirow{6}{*}{ girder } & Young modulus of elasticity & $E=210 \times 10^{9} \mathrm{~N} / \mathrm{m}^{2}$ \\
& shear modulus of elasticity & $G=80.8 \times 10^{9} \mathrm{~N} / \mathrm{m}^{2}$ \\
& element length & $L=40 \mathrm{~m}$ \\
& half chord width of the deck & $b=15.5 \mathrm{~m}$ \\
& girder mass & $m_{g}=15260 \mathrm{~kg} / \mathrm{m}$ \\
& moment of inertia about horizontal axis & $I_{y}=4 \mathrm{~m}^{4}$ \\
& moment of inertia about vertical axis & $I_{z}=100 \mathrm{~m}^{4}$ \\
& torsional moment of inertia & $I_{a}=7.6 \mathrm{~m}^{4}$ \\
& mass moment of inertia (including cables) & $I_{m}=2.47 \times 10^{6} \mathrm{kgm}^{2} / \mathrm{m}$ \\
\hline \multirow{4}{*}{ cables } & $E_{c}=210 \times 10^{9} \mathrm{~N} / \mathrm{m}^{2}$ \\
& Young modulus of elasticity & $A_{c}=0.44 \mathrm{~m}^{2}$ \\
& cross sectional area & $b_{c}=13.5$ \\
& half chord distance between cables & $d_{c}=0.75 \mathrm{~m}$ \\
& diameter & $m_{c}=2 \times 3745 \mathrm{~kg} / \mathrm{m}$ \\
& mass of the main cables (both cables) & $H_{w}=2 \times 1.92 \times 10^{8} \mathrm{~N}$ \\
& horizontal cable tension (both cables) & $f=180 \mathrm{~m}$ \\
& main cable sag & $h_{e, \text { min }}=10 \mathrm{~m}$ \\
& length of shortest hanger cable &
\end{tabular}

the downward movement on one side of the centreline of the centre span tends to increase the cable length, while at the same time the upward movement on the other side of the centre span tends to reduce the cable length, and the effects balance each other. In consequence of the lack of additional cable tension there is not interaction between the main and side spans for the antisymmetric modes

\begin{tabular}{|c|c|c|c|}
\hline vertical modes [18] & FE vertical modes & lateral modes [18] & FE lateral modes \\
\hline V. mode 1: $0.100 \mathrm{~Hz}$ & V. mode 1:0.096 Hz & 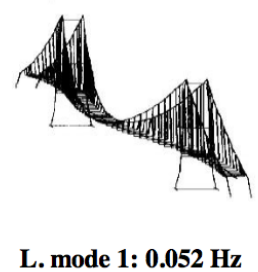 & L. mode 1: $0.064 \mathrm{~Hz}$ \\
\hline V. mode 2: $0.115 \mathrm{~Hz}$ & V. mode 2: $0.089 \mathrm{~Hz}$ & L. mode 2: $0.123 \mathrm{~Hz}$ & L. mode 2: $0.112 \mathrm{~Hz}$ \\
\hline V. mode 3: $0.135 \mathrm{~Hz}$ & V. mode 3: $0.133 \mathrm{~Hz}$ & L. mode 3: $0.187 \mathrm{~Hz}$ & L. mode 3: $0.189 \mathrm{~Hz}$ \\
\hline
\end{tabular}

Fig. 3: Comparison of experimentally identified vertical and lateral modes to FE results. 
due to the cables and any movement of the side spans is due to the continuous girder assumption. In general the structural model captures the necessary vertical and torsional modal characteristics of the structure which are fundamental for the flutter instability analysis.

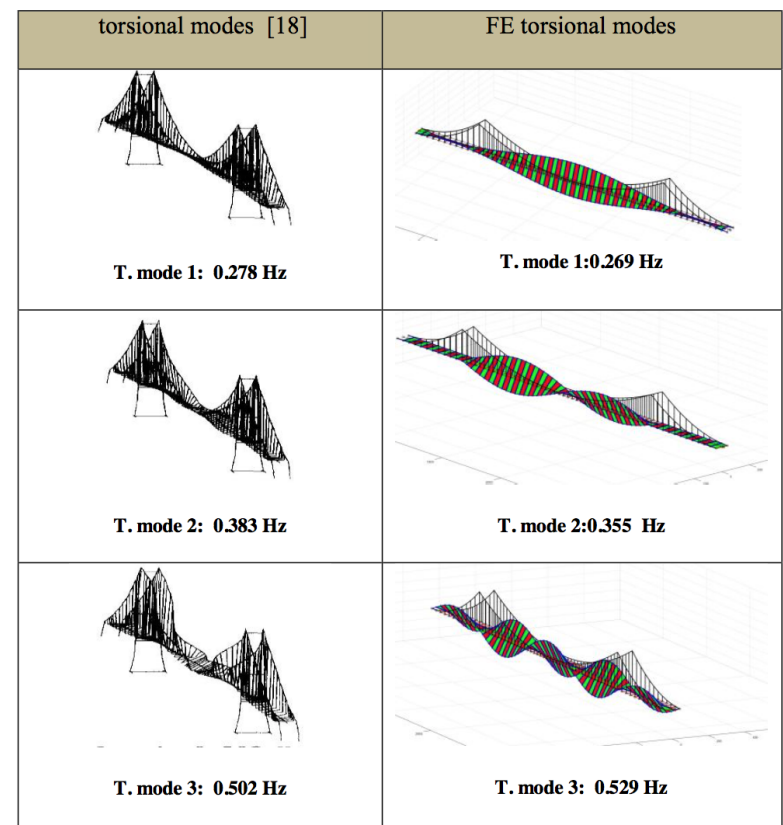

Fig. 4: Comparison of experimentally identified torsional modes to FE results.

\section{Aeroelastic Models}

In this section we develop the FE aeroelastic model using classical thin aerofoil theory as well as the flutter derivatives approach.

\section{A. Sectional Model with Thin Aerofoil Theory}

The lift $L_{s e}$ and moment $M_{s e}$ expressions presented for a thin aerofoil are taken from Theodorsen's original paper [39]:

$$
\begin{aligned}
L_{s e} & =-\pi \rho b^{2}[U \dot{\alpha}+\ddot{h}]-2 \pi \rho U b C(k)\left[U \alpha+\dot{h}+\frac{\dot{\alpha} b}{2}\right] \\
M_{s e} & =-\pi \rho b^{2}\left[\frac{b U \dot{\alpha}}{2}+\frac{b^{2} \ddot{\alpha}}{8}\right]+\pi \rho U b^{2} C(k)\left[U \alpha+\dot{h}+\frac{b \dot{\alpha}}{2}\right]
\end{aligned}
$$

where $\rho$ is the mass of air per unit volume, $b$ is the half chord of the wing, $U$ is the wind velocity and $C(k)$ represents the Theodorsen circulation function:

$$
C(k)=\frac{J_{1}(k)-i Y_{1}(k)}{\left(J_{1}(k)+Y_{0}(k)\right)-i\left(Y_{1}(k)-J_{0}(k)\right)}
$$

in which $J_{0}(k), J_{1}(k), Y_{0}(k), Y_{1}(k)$ are Bessel functions of the first and second kind respectively, $k=$ $\omega b / U$ is the reduced frequency and $i=\sqrt{-1}$. The equations of motion of the sectional model (2DOF aerofoil with heave and pitch) combined with the aerodynamic forces given in Eq.(3),(4) can be expressed in the following state space form: 


$$
\left[\begin{array}{cc}
0 & M-M_{n c} \\
I & 0
\end{array}\right]\left[\begin{array}{c}
\dot{q} \\
\dot{q}_{v}
\end{array}\right]=\left[\begin{array}{cc}
-K & -C+C_{n c} \\
0 & I
\end{array}\right]\left[\begin{array}{c}
q \\
q_{v}
\end{array}\right]+\Xi_{C}
$$

where $\Xi_{C}$ represents the circulatory terms and

$$
\begin{aligned}
M=\left[\begin{array}{cc}
m & 0 \\
0 & I_{m}
\end{array}\right] \quad C & =\left[\begin{array}{cc}
2 \omega_{h} m \zeta_{h} & 0 \\
0 & 2 \omega_{a} I_{m} \zeta_{a}
\end{array}\right] \quad K=\left[\begin{array}{cc}
\omega_{h}^{2} m & 0 \\
0 & \omega_{a}^{2} I_{m}
\end{array}\right] \\
M_{n c} & =\left[\begin{array}{cc}
-\pi \rho b^{2} & 0 \\
0 & -\frac{\pi \rho b^{4}}{8}
\end{array}\right] \quad C_{n c}=\left[\begin{array}{cc}
0 & -\pi \rho b^{2} U \\
0 & -\frac{\pi \rho b^{3} U}{2}
\end{array}\right]
\end{aligned}
$$

with $\omega_{h}, \omega_{a}$ the natural frequencies for the vertical/heave and torsional/pitch modes of the structure, $\zeta_{h}, \zeta_{a}$ the corresponding critical damping ratios, $q$ the position vector defined as $q=\{h, \alpha\}^{T}$ and $q_{v}$ the velocity vector defined as $q_{v}=\{\dot{h}, \dot{\alpha}\}^{T}$. When introducing the state vector $x=\left\{q, q_{v}\right\}^{T}$, Eq.(6) can be expressed in the form:

$$
E_{c} \dot{x}=A_{c} x+\Xi_{C}
$$

where

$$
E_{c}=\left[\begin{array}{cc}
0 & M-M_{n c} \\
I & 0
\end{array}\right] \quad A_{c}=\left[\begin{array}{cc}
-K & -C+C_{n c} \\
0 & I
\end{array}\right]
$$

Matrices $M_{n c}, C_{n c}$ represent the non-circulatory part of the aerodynamic forces and are usually referred to as aerodynamic (or added) mass and damping matrices.

The circulatory term $\Xi_{C}$ depends on the irrational function $C(k)$ and therefore cannot be expressed in a state space form. As a consequence the traditional approach to aeroelastic stability analysis is based on finding iteratively wind speed(s) for which sinusoidal solutions to Eq.(9) (or alternatively Eq.(6)) exist. Standard algorithms, including the k-method and the (p-k)-method [7], require the repeated evaluation of the circulation function $C(k)$ in Eq.(5), which is a combination of Bessel functions. This approach has several disadvantages. First, one must seek out the resonant frequencies one at a time (one of which might be zero). Second, one must distinguish between single and coupled-mode cases - this becomes particularly inconvenient when high-order modal combinations are possible. Third, during a design exercise, one cannot form a clear picture as to how design changes are influencing the aeroelastic stability of the structure as a whole, which may possibly include multiple flutter modes as well as multiple divergence modes [13]. It is thus convenient to approximate the circulation function with a low-order rational function. Approximations of this type allow one to write the problem in a state space form and to replace the aforementioned methods with classic-theoretic analytic devices such as root-locus diagrams. By invoking a least squares approximation an accurate quartic approximation of $C(k)$ was found:

$$
C(k)=\frac{0.995+57.018(i k)+23.788(i k)^{2}+1895.463(i k)^{3}+1523.247(i k)^{4}}{1+62.304(i k)+807.784(i k)^{2}+3060.678(i k)^{3}+3033.763(i k)^{4}}
$$

The Nyquist diagram of the Theodorsen circulation function, the often-used Jones approximation [18] and the here used quartic approximation are shown in Fig.5(a). Clearly the quartic approximation is accurate with the lower order Jones function somewhat less so. Finally, employing the concepts of analytic continuation, $i k$ in Eq.(11) can be replaced with $\hat{s}=s b / U$, i.e. the expression derived for oscillatory motion can be extended to use with arbitrary motion. 


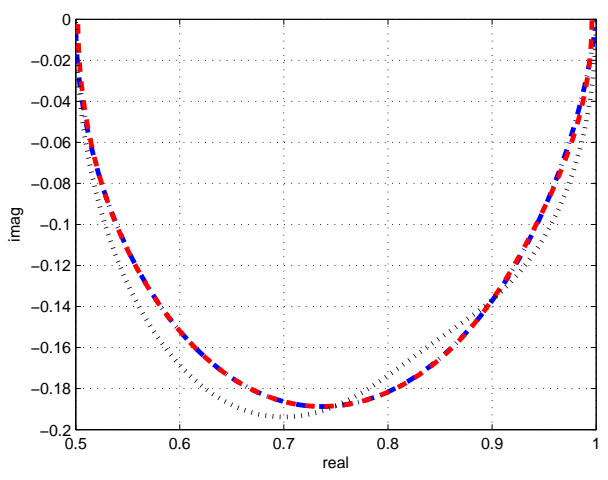

(a)

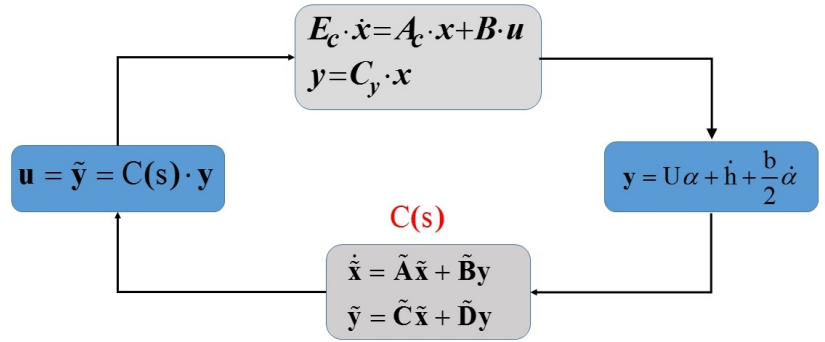

(b)

Fig. 5: (a) The Theodorsen function $C(k)$ and its rational approximations: the exact function is the (blue) dot-dash curve, the quartic approximation the (red) dashed curve, and the Jones approximation the (black) dotted curve. (b) Block diagram of the open loop aerodynamic system of a 2 DOF sectional model.

The circulatory term $\Xi_{C}$ of the aerodynamic forces can be expressed mathematically by the feedbackloop system in Fig.5(b), in which the approximation of the circulation function $C(s)$ can be viewed as a transfer function that receives the structural response $y=U \alpha+\dot{h}+\dot{\alpha} b / U$ as input and generates the output $\tilde{y}$, which is finally translated into lift and moment by the factors $-2 \pi \rho U b$ and $\pi \rho U b^{2}$. An equivalent state space realization $\tilde{A}, \tilde{B}, \tilde{C}, \tilde{D}$ of $C(s)$ is derived using standard techniques. Note that the number of (aerodynamic) states of such state space is four, because $C(s)$ is forth order.

The open loop system dynamics for the sectional model including the circulatory terms are derived by augmenting the state space in Eq.(9) to include the aerodynamic states related to the circulatory function:

$$
E \dot{\hat{x}}=A \hat{x}
$$

where

$$
E=\left[\begin{array}{cc}
E_{c} & 0 \\
0 & I
\end{array}\right] \quad A=\left[\begin{array}{cc}
A_{c}+B \tilde{D} C_{y} & B \tilde{C} \\
\tilde{B} C_{y} & \tilde{A}
\end{array}\right] \quad B=\left[\begin{array}{c}
-2 \pi \rho U b \\
\pi \rho U b^{2} \\
0 \\
0
\end{array}\right] \quad C_{y}=\left[\begin{array}{llll}
0 & U & 1 & b / 2
\end{array}\right]
$$

with the matrices $B$ and $C_{y}$ explicitly derived from Eqns.(3),(4) and $\hat{x}$ consisting of the four 'structural' states (heave, pitch, and related velocities) plus the four 'aerodynamic'. The eigenvalues of Eq.(12) as a function of the wind speed $U$ will determine the stability of the open-loop system. More specifically the wind speed at which an eigenvalue has positive real part signifies the onset of an instability.

\section{B. Finite Element Model with Thin Aerofoil Theory}

In order to include the aeroelastic forcing in a general FE formulation procedure, distributed lifting loading and distributed moments are considered for every element. The procedure followed here assumes lumping of the distributed forces on the nodes. The aerodynamic mass and damping matrices are now of dimension $12 \times 12$ with the non-zero entries corresponding to the heave and pitch related 
degrees of freedom and can be superimposed at the corresponding degrees of freedom in the same way as the structural matrices:

$$
M_{a}=\sum_{i=1}^{N} M_{n c, i} \quad C_{a}=\sum_{i=1}^{N} C_{n c, i}
$$

The equations of motion in a FE format now take the following form, similar to the case with 2-DOF sectional model in Eq.(6):

$$
\left[\begin{array}{cc}
0 & M-M_{a} \\
I & 0
\end{array}\right]\left[\begin{array}{c}
\dot{Q} \\
\dot{Q}_{v}
\end{array}\right]=\left[\begin{array}{cc}
-K & -C+C_{a} \\
0 & I
\end{array}\right]\left[\begin{array}{c}
Q \\
Q_{v}
\end{array}\right]+\Xi_{C}
$$

where $Q$ is a vector containing the degrees of freedom of the bridge structure, $Q_{v}$ the related velocities, and the state vector is defined as $\hat{x}=\left\{Q, Q_{v}\right\}^{T}$. We then construct a feedback system with as many feedback loops as the number of elements used, Fig.10. In a similar manner as in the sectional model of Eq.(12), the finite element system dynamics are expressed in the form:

$$
E_{g} \dot{\hat{x}}=A_{g} \hat{x}
$$

where

$$
E_{g}=\left[\begin{array}{cc}
E_{c} & 0 \\
0 & I
\end{array}\right] \quad A_{g}=\left[\begin{array}{cc}
A_{c}+B_{g} \widetilde{D}_{g} C_{g} & B_{g} \widetilde{C}_{g} \\
\widetilde{B}_{g} C_{g} & \widetilde{A}_{g}
\end{array}\right]
$$

in which $\tilde{A_{g}}=\operatorname{diag}(\tilde{A}), \tilde{B_{g}}=\operatorname{diag}(\tilde{B}), \tilde{C_{g}}=\operatorname{diag}(\tilde{C}), \tilde{D_{g}}=\operatorname{diag}(\tilde{D})$ and $\hat{x}$ consists of the structural states, their velocities and the aerodynamic states related to the circulatory functions (namely there are four aerodynamic states for each of the finite element of the model, because there is a circulatory function for each element).

\section{Great Belt Bridge with Thin Aerofoil Theory}

The Great Belt Bridge was again used in order to implement the aeroelastic FE procedure. The structural damping matrix is assessed by assuming a Rayleigh proportional damping model $C=$ $a_{M} M+a_{K} K$. The parameters $a_{M}, a_{K}$ are chosen so as to result in a $1 \%$ critical damping ratio for the first vertical and torsional mode. Fig.6 presents the open loop root locus diagram of the Great Belt Bridge, in which the wind velocity $U$ is the varied parameter. From the diagram one can observe two instability modes, which cross into the real positive plane of the calculated eigenvalues. One presents itself as a complex pair of eigenvalues, and corresponds to an oscillatory divergent motion (flutter), whereas the other has real eigenvalues and consequently presents a monotonically increasing instability due to steady-state aerodynamic moment. As shown in Fig.6 the Great Belt Bridge model reaches flutter instability at $82 \mathrm{~m} / \mathrm{s}$ and torsional divergence at $90 \mathrm{~m} / \mathrm{s}$.

Flutter speed estimates for the Great Belt Bridge have been reported elsewhere [19] but these predictions have a large scatter, ranging from 62 to $97 \mathrm{~m} / \mathrm{s}$, see Tab.II, which gives a summary of these results as well as the values predicted by the proposed analytical framework. Flutter speeds based on an equivalent 2-DOF, heave-pitch model, both using thin aerofoil theory and flutter derivatives are included. The predicted flutter speed using the 2-DOF model is the same as Larsen analytic flutter prediction using flutter derivatives (measured on a sectional model)[21], which was based on a two mode routine with allowance for the mode shapes of the basic symmetric bending and torsion mode. The higher critical wind speeds obtained with the full structural modes can be attributed to multimodal behaviour with higher vertical modes participating in the first flutter mode as will be discussed in $\S \mathrm{V}$. 


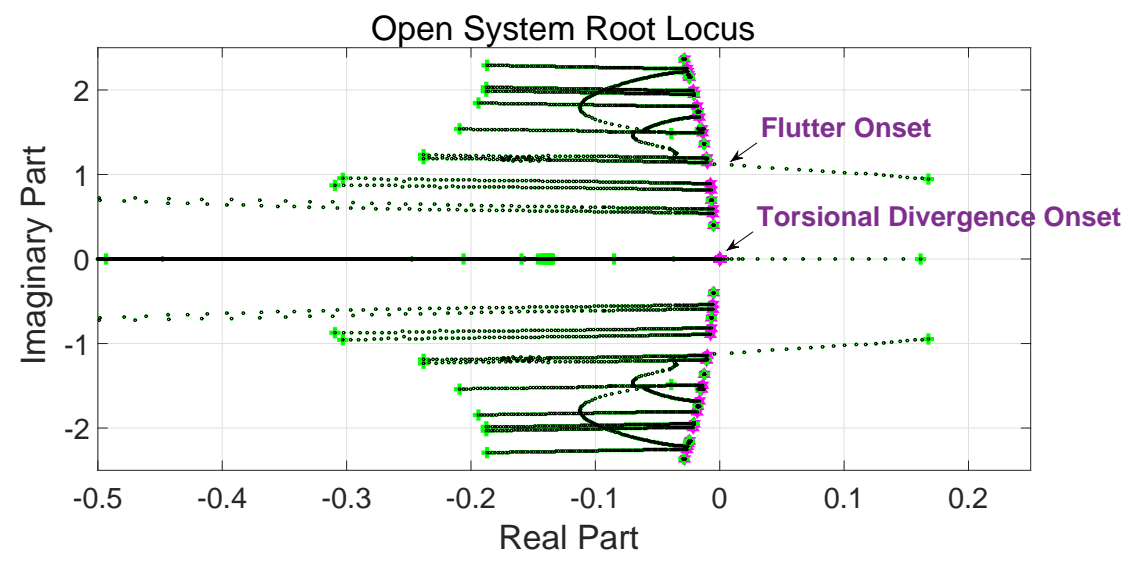

Fig. 6: Root-locus of the Great Belt Bridge. The wind speed is swept from 0 (magenta hexagon) to $100 \mathrm{~m} / \mathrm{s}$ (green cross). The flutter mode goes unstable at $82 \mathrm{~m} / \mathrm{s}$ while the torsional divergence mode goes unstable at $90 \mathrm{~m} / \mathrm{s}$.

TABLE II: Estimation of flutter velocities of the Great Belt Bridge from literature.

\begin{tabular}{ll}
\hline Reference & Flutter Velocity \\
\hline [19]: numerical, flutter derivatives, 2 modes & $90 \mathrm{~m} / \mathrm{s}$ \\
[19]: numerical, flutter derivatives, 18 modes & $62 \mathrm{~m} / \mathrm{s}$ \\
[10]: numerical, flutter derivatives, 2 modes & $83 \mathrm{~m} / \mathrm{s}$ \\
[10]: numerical, flutter derivatives, 6 modes & $97 \mathrm{~m} / \mathrm{s}$ \\
[21]: wind tunnel section model & $70-74 \mathrm{~m} / \mathrm{s}$ \\
[21]: wind tunnel taut strip model & $72 \mathrm{~m} / \mathrm{s}$ \\
[21]: wind tunnel full bridge model & $70-75 \mathrm{~m} / \mathrm{s}$ \\
[21]: numerical, flutter derivatives from section model & $75 \mathrm{~m} / \mathrm{s}$ \\
[21]: numerical, flutter derivatives from taut strip model & $79 \mathrm{~m} / \mathrm{s}$ \\
this work: numerical, flutter derivatives, all modes & $81 \mathrm{~m} / \mathrm{s}$ \\
this work: numerical, thin aerofoil, all modes & $82 \mathrm{~m} / \mathrm{s}$ \\
this work: numerical, flutter derivatives, 2 modes & $75 \mathrm{~m} / \mathrm{s}$ \\
this work: numerical, thin aerofoil, 2 modes & $75 \mathrm{~m} / \mathrm{s}$ \\
\hline
\end{tabular}

\section{Finite Element Model with Flutter Derivatives}

Theodorsen's approach of using velocity potentials due to the air flow around the deck and adoption of the Kutta condition [24] may result in significant errors if the deck girder is considered to fall into the bluff body category for this reason the use of the so-called "Flutter Derivatives" was introduced by Scanlan [34]. There are several different formulations, using different combinations of $k, K, b, B$. In this work we adopt the following notation:

$$
\begin{aligned}
& L_{s e}=\frac{1}{2} \rho U^{2} B\left[K H_{1}^{*}\left(\frac{\dot{h}}{U}\right)+K H_{2}^{*} B\left(\frac{\dot{\alpha}}{U}\right)+K^{2} H_{3}^{*} \alpha+K^{2} H_{4}^{*}\left(\frac{h}{B}\right)\right] \\
& M_{s e}=\frac{1}{2} \rho U^{2} B^{2}\left[K A_{1}^{*}\left(\frac{\dot{h}}{U}\right)+K A_{2}^{*} B\left(\frac{\dot{\alpha}}{U}\right)+K^{2} A_{3}^{*} \alpha+K^{2} A_{4}^{*}\left(\frac{h}{B}\right)\right]
\end{aligned}
$$

where $H_{1}^{*}, A_{1}^{*}$ are the dimensionless flutter derivatives, which are a function of the reduced frequency $K=\omega B / U, \omega$ is the circular frequency and $B=2 b$ is the deck width. In this formulation the drag degree of freedom is omitted since our aim is to compare this procedure to the thin aerofoil theory. However the drag effect has been shown to be important for certain cases like for the truss-decked Akashi-Kaikyo Bridge, the world's longest suspension bridge, [33]. The theoretical values of the flutter derivatives of a thin aerofoil can be computed in terms of the Theodorsen circulation function giving the following expressions [32]: 


$$
\begin{array}{cc}
H_{1}^{*}=-2 \pi F(k) / K & A_{1}^{*}=\pi F(k) / 2 K \\
H_{2}^{*}=-\frac{\pi}{2 K}\left[1+\frac{4 G(k)}{K}+F(k)\right] & A_{2}^{*}=-\frac{\pi}{2}\left[\frac{K}{4}-G(k)-\frac{K F(k)}{4}\right] \\
H_{3}^{*}=-\frac{\pi}{K^{2}}\left[2 F(k)-\frac{1}{2} G K\right] & A_{3}^{*}=\frac{\pi}{2 K^{2}}\left[\frac{K^{2}}{32}+F(k)-\frac{K G(k)}{4}\right] \\
H_{4}^{*}=K^{2}\left[1+\frac{4 G}{K}\right] & A_{4}^{*}=-\pi G(k) / 2 K
\end{array}
$$

where $K=2 k=2 \omega b / U$ and $F(k), G(k)$ are the real and imaginary part of the Theodorsen function $C(k)=F(k)+i G(k)$. Eqns.(18),(19) can be rewritten in a matrix form:

$$
\begin{aligned}
\left\{\begin{array}{c}
L_{s e} \\
M_{s e}
\end{array}\right\}= & \frac{1}{2} \rho U^{2} B\left[\begin{array}{cc}
\frac{K H_{1}^{*}}{U} & K H_{2}^{*} \frac{B}{U} \\
\frac{B K A_{1}^{*}}{U} & B K A_{2}^{*} \frac{B}{U}
\end{array}\right]\left\{\begin{array}{c}
\dot{h} \\
\dot{\alpha}
\end{array}\right\} \\
& +\frac{1}{2} \rho U^{2} B\left[\begin{array}{cc}
\frac{K^{2} H_{4}^{*}}{B} & K^{2} H_{3}^{*} \\
K^{2} A_{4}^{*} & K^{2} A_{3}^{*} B
\end{array}\right]\left\{\begin{array}{c}
h \\
\alpha
\end{array}\right\}
\end{aligned}
$$

Eq.(21) can now be expressed in the frequency domain $(\dot{h}=i \omega h, \dot{\alpha}=i \omega \alpha)$ and the RFA $\hat{Q}(i K)$ of the resulting matrix can be introduced:

$$
\begin{aligned}
\left\{\begin{array}{c}
L_{s e} \\
M_{s e}
\end{array}\right\} & =\frac{1}{2} \rho U^{2}\left[\begin{array}{cc}
K^{2} H_{4}^{*}+i K^{2} H_{1}^{*} & B\left[K^{2} H_{3}^{*}+i K^{2} H_{2}^{*}\right] \\
B\left[K^{2} A_{4}^{*}+i K^{2} A_{1}^{*}\right] & B^{2}\left[K^{2} A_{3}^{*}+i K^{2} A_{2}^{*}\right]
\end{array}\right]\left\{\begin{array}{l}
h \\
\alpha
\end{array}\right\} \\
& =U^{2} \cdot \hat{Q}(i K) \cdot\left\{\begin{array}{c}
h \\
\alpha
\end{array}\right\}
\end{aligned}
$$

in this work the so-called Roger's approximation [31] is employed:

$$
\hat{Q}(i K)=P_{0}+P_{1} \cdot i K+P_{2} \cdot(i K)^{2}+\sum_{j=3}^{N} \frac{P_{j} \cdot i K}{i K+\gamma_{j-2}}
$$

where the matrices $P_{0}, P_{1}, P_{2}, P_{j}$ and the coefficients $\gamma_{j-2}$ are frequency independent, and are found by fitting against the wind tunnel experimental data of the deck section model. Note that $P_{0}, P_{1}, P_{2}$ are the non-circulatory stiffness, damping and inertia (added-mass) while $P_{j}, \gamma_{j-2}$ are related to the circulatory forces. In the present work we use four poles, i.e. $N=4$. Similarly to the case of thin aerofoil theory, $i K$ in Eq.(23) can be replaced with $\hat{s}=s B / U$.The equations of motion for the sectional model can now be rewritten in the Laplace domain as:

$$
\begin{aligned}
\left(M s^{2}+C s+K\right) q(s) & =U^{2} \hat{Q}(\hat{s}) q(s) \\
& =U^{2}\left(P_{0}+P_{1} s \frac{B}{U}+P_{2} s^{2}\left(\frac{B}{U}\right)^{2}+\sum_{j=3}^{N} \frac{P_{j} \cdot s \frac{B}{U}}{s^{B}+\gamma_{j-2}}\right) q(s)
\end{aligned}
$$

Eq.(24) is converted back to the time domain by taking the inverse Laplace transform. In particular note that the inverse Laplace transform of the circulatory terms (normalized by $U^{2} P_{j}$ ) reads:

$$
X_{a j}(s)=\frac{s \frac{B}{U}}{s \frac{B}{U}+\gamma_{j-2}} q(s) \stackrel{\mathcal{L}^{-1}}{\longrightarrow} \dot{X}_{a j}(t)=\dot{q}(t)-\frac{U}{B} \gamma_{j-2} X_{a j}(t)
$$

where $X_{a j}(t)$ is the aerodynamic state associated with the circulatory term which adds the structural states $q$. Therefore the system equations in the time domain are: 


$$
\left\{\begin{array}{c}
\dot{q} \\
\ddot{q} \\
\dot{X}_{a 3} \\
\dot{X}_{a 4} \\
\dot{X}_{a 5} \\
\dot{X}_{a 6}
\end{array}\right\}=\left[\begin{array}{cccccc}
0 & I & 0 & 0 & 0 & 0 \\
-\bar{M}^{-1} \bar{K} & -\bar{M}^{-1} \bar{C} & U^{2} \bar{M}^{-1} P_{3} & U^{2} \bar{M}^{-1} P_{4} & U^{2} \bar{M}^{-1} P_{5} & U^{2} \bar{M}^{-1} P_{6} \\
0 & I & -\left(\frac{U}{B}\right) \gamma_{1} I & 0 & 0 & 0 \\
0 & I & 0 & -\left(\frac{U}{B}\right) \gamma_{2} I & 0 & 0 \\
0 & I & 0 & 0 & -\left(\frac{U}{B}\right) \gamma_{3} I & 0 \\
0 & I & 0 & 0 & 0 & -\left(\frac{U}{B}\right) \gamma_{4} I
\end{array}\right]\left\{\begin{array}{c}
q \\
\dot{q} \\
X_{a 3} \\
X_{a 4} \\
X_{a 5} \\
X_{a 6}
\end{array}\right\}(26)
$$

where $\bar{M}=M-U^{2} P_{2}\left(\frac{b}{U}\right)^{2}, \bar{C}=C-U^{2} P_{1}\left(\frac{b}{U}\right), \bar{K}=K-U^{2} P_{0}$.

Similar to the case of thin aerofoil theory, the flutter derivatives approach can be extended to a FE form, where again the lift and moment equations Eq.(18),(19) are lumped at the end nodes of every element. For the FE model the elemental matrices $P_{0 e}, P_{1 e}, P_{2 e}$ related to the noncirculatory components have dimensions $12 \times 12$ and can be superimposed similarly to the structural matrices:

$$
P_{0 G}=\sum_{i=1}^{N} P_{0 e, i} \quad P_{1 G}=\sum_{i=1}^{N} P_{1 e, i} \quad P_{2 G}=\sum_{i=1}^{N} P_{2 e, i}
$$

where the summation is again intended in the FE sense. Since the aerodynamic states $X_{a j}, j=3 . .6$ are related to the pitch and heave of every node, the matrices $P_{j e}, j=3 . .6$ have size $12 \times 4$ :

$$
P_{j}=\left(\begin{array}{cc}
P_{j}^{11} & P_{j}^{12} \\
P_{j}^{21} & P_{j}^{22}
\end{array}\right) \rightarrow P_{j e}=\frac{L}{2}\left(\begin{array}{cccc}
P_{\text {heave }}^{P_{j}^{11}} & P_{j}^{12} & 0 & 0 \\
\vdots & \vdots & \vdots & \vdots \\
P_{j}^{21} & \underbrace{P_{j}^{22}}_{\text {pitch }} & 0 & 0 \\
0 & 0 & \underbrace{P_{j}^{11}}_{\text {heave }} & P_{j}^{12} \\
& \vdots & \vdots & \vdots \\
\vdots & 0 & P_{j}^{21} & \underbrace{P_{j}^{22}}_{\text {pitch }}
\end{array}\right)
$$

It is worth noting at this stage that this formulation introduces 8 extra aerodynamic states per node (four related to the pitch and four related to the heave, because the flutter derivatives were approximated using four poles) as opposed to the case of thin aerofoil which introduces just 4 (related to the four poles used to approximate the Theodorsen function).

\section{E. Application and Comparison of Flutter Derivatives to the Great Belt Bridge}

We implement the procedure described in §III-D for the experimental data obtained at the water channel of the University of Hamburg, reported in Starossek et al. [38] and later in [24]. Fig.7 presents the approximation for the four terms of the matrix $\hat{Q}$ in Eq.(23) when thin aerofoil theory and experimental derivatives from the Great Belt are used. The RFA as shown in Fig.7(a) results in a very good fit when thin aerofoil theory is used since the points fall on smooth lines. For the experimental data irregularities pose difficulties in fitting but still the results are reasonably good, Fig.7(b). These can of course be improved with an increasing number of aerodynamic poles which in turn though increases the dimensions of the state space. A non-linear least square procedure was also implemented in the approximation where the location of the poles was not preselected but optimized: 
however this yielded only a marginally better improvement of the fitting.

Fig. 8 presents a comparison of the root-loci of the full bridge when using thin aerofoil theory, namely Fig.6, and flutter derivatives. The similarity of aerodynamic behaviour and the assumption that the streamlined box girder under investigation resembles the behaviour of a thin aerofoil is apparent. Both thin aerofoil theory and the use of flutter derivatives are very close in their prediction of the flutter critical wind speed $(81 \mathrm{~m} / \mathrm{s}$ vs. $82 \mathrm{~m} / \mathrm{s})$.

The lift and moment on the system including leading- and trailing-edge flaps was based on a transformation of the wing-aileron-tab configuration [40]. The transformation is depicted in Fig.9. In this new configuration the aileron becomes the bridge deck, the wing is the leading-edge flap and the tab the trailing-edge-flap. In order to re-level the bridge, and return its mass centre to the correct position, pitch and heave correction must be applied. For the system depicted in Fig.9 the inertial properties of the flaps were neglected and only their aerodynamic influence on the lift and moment applied on the deck was considered. This is mathematically simplifying assumption based on the premise that the mass of the flaps should be kept considerably smaller that the mass of the deck in
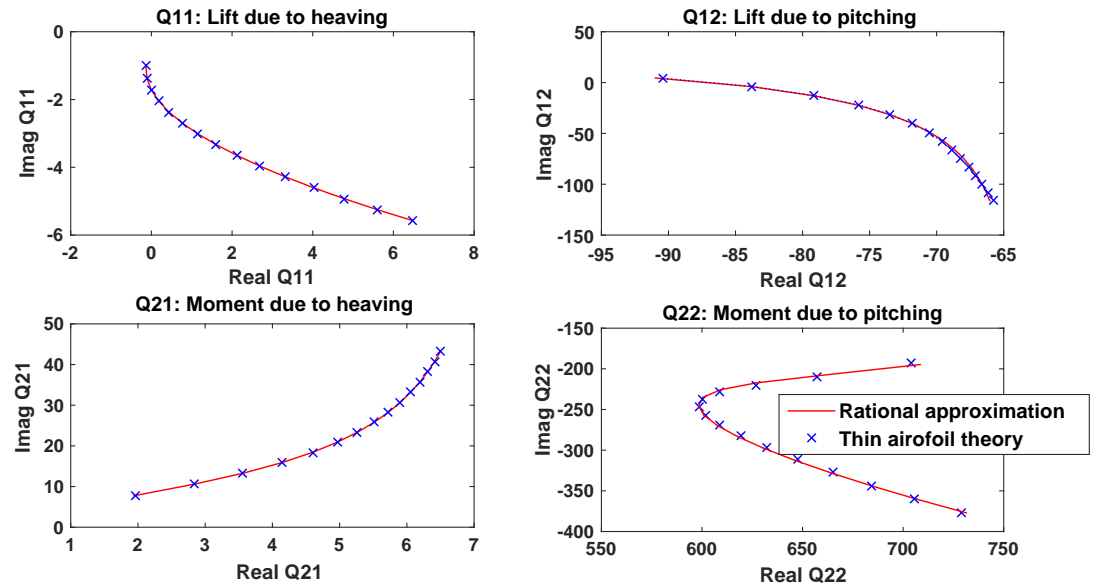

(a)
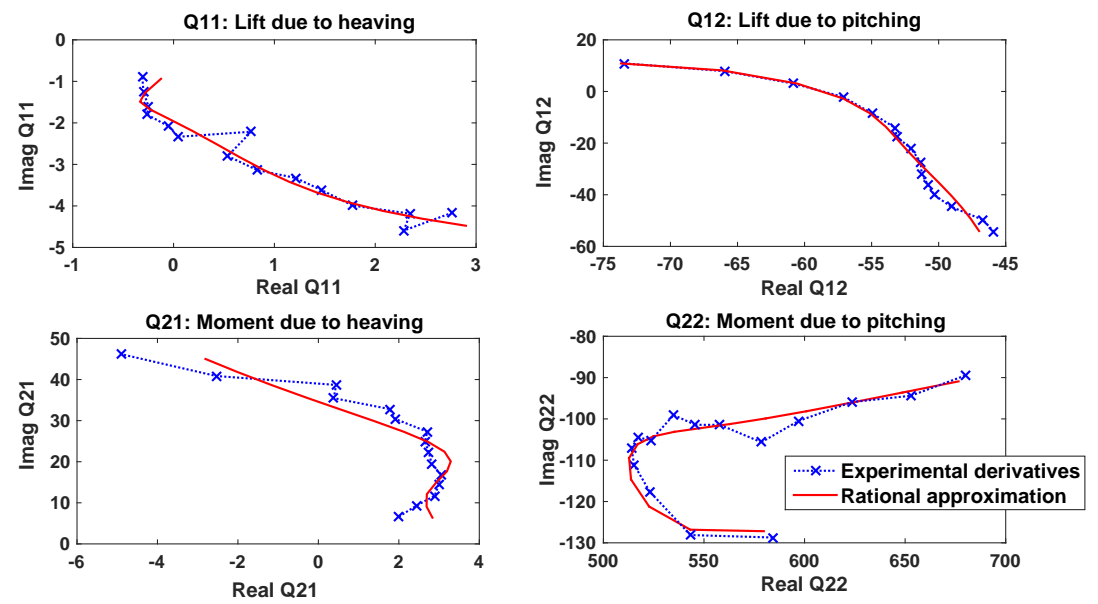

(b)

Fig. 7: Roger's approximation for the four terms of $\hat{Q}$ when thin aerofoil (a) and experimental flutter derivatives (b) are implemented. 


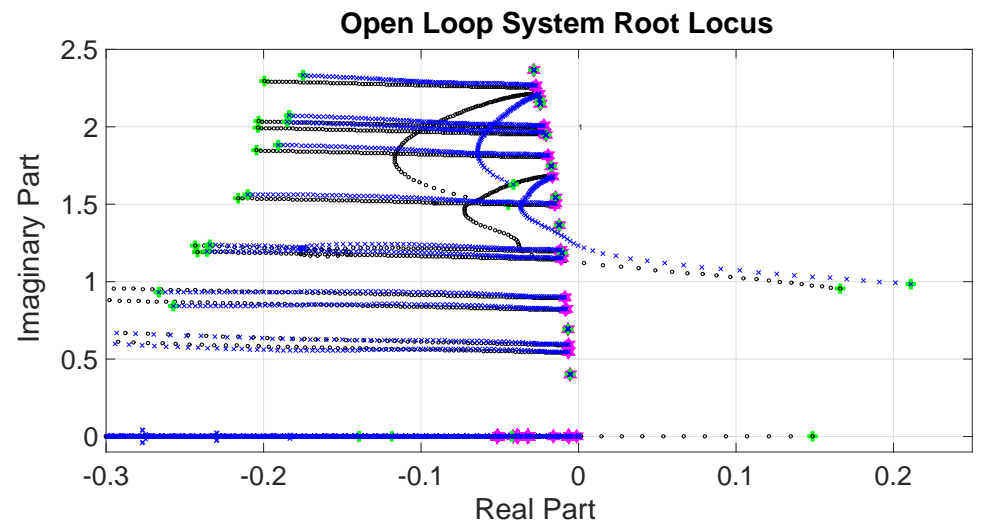

Fig. 8: Comparison of root-loci for the Great Belt Bridge using thin aerofoil theory and flutter derivatives. The wind is swept from 0 (magenta hexagon) to $100 \mathrm{~m} / \mathrm{s}$ (green cross) The black circles correspond to the use of thin aerofoil theory and the blue crosses to the use of flutter derivatives. Thin aerofoil theory predicts flutter at $82 \mathrm{~m} / \mathrm{s}$ and flutter derivatives at $81 \mathrm{~m} / \mathrm{s}$.

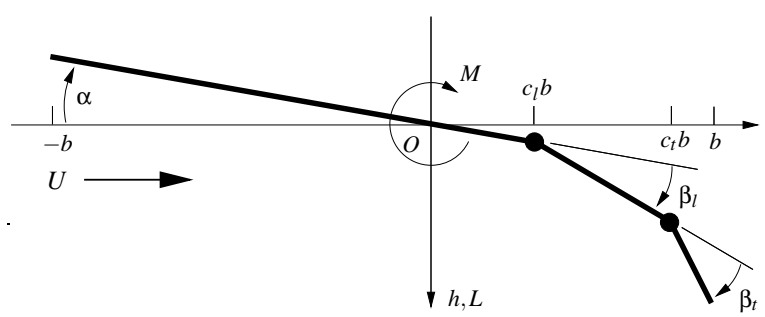

(A)

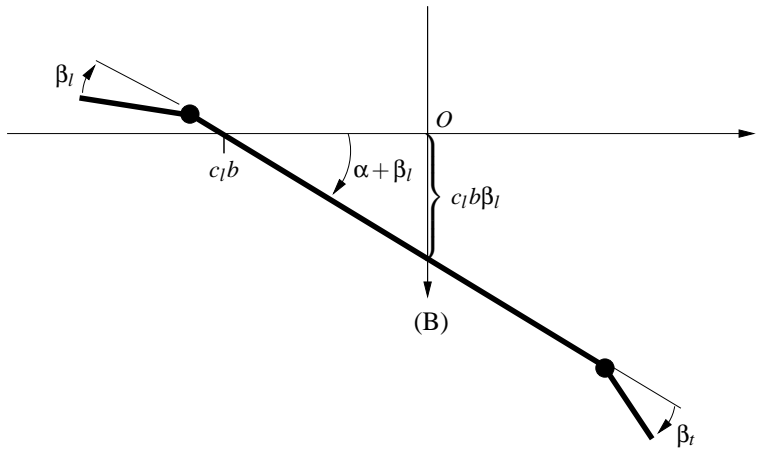

Fig. 9: Transformation of the Theodorsen-Garrick wing-aileron-tab configuration into a controlled bridge deck. (A): The wing pitch angle is $\alpha$, the aileron angle is $\beta_{l}$ and the tap angle is $\beta_{t}, U$ denotes the wind speed. (B): The leading flap angle is $\beta_{l}$, the deck angle is $\alpha$ and the trailing-edge flap angle is $\beta_{t}$.

order to minimize the additional dead load. In a similar manner as for the case of sole deck Eq.(12) holds, with the aerodynamic mass, damping and stiffness matrices are formed as follows: 


$$
\begin{aligned}
\mathrm{M}_{n c F}= & {\left[\begin{array}{cccc}
-\pi \rho b^{2} & 0 & M_{n c F}^{1,3} & M_{n c F}^{1,4} \\
0 & -\frac{\pi \rho b^{4}}{8} & M_{n c F}^{2,3} & M_{n c F}^{2,4} \\
0 & 0 & 0 & 0 \\
0 & 0 & 0 & 0
\end{array}\right] } \\
\mathrm{C}_{n c F}= & {\left[\begin{array}{cccc}
0 & -\pi \rho b^{2} U & C_{n c F}^{1,3} & C_{n c F}^{1,4} \\
0 & -\frac{\pi}{2} \rho b^{3} U & C_{n c F}^{2,3} & C_{n c F}^{2,4} \\
0 & 0 & 0 & 0 \\
0 & 0 & 0 & 0
\end{array}\right] } \\
\mathrm{K}_{n c F}= & {\left[\begin{array}{cccc}
0 & 0 & K_{n c F}^{1,3} & K_{n c F}^{1,4} \\
0 & 0 & K_{n c F}^{2,3} & K_{n c F}^{2,4} \\
0 & 0 & 0 & 0 \\
0 & 0 & 0 & 0
\end{array}\right] }
\end{aligned}
$$

where

$$
\begin{aligned}
M_{n c F}^{1,3} & =\rho b^{3} T_{1}\left(c_{t}\right) \\
M_{n c F}^{1,4} & =\rho b^{3}\left(T_{1}\left(c_{l}\right)-\pi c_{l}\right) \\
M_{n c F}^{2,3} & =\rho b^{4}\left(T_{7}\left(c_{t}\right)+c_{t} T_{1}\left(c_{t}\right)\right) \\
M_{n c F}^{2,4} & =\rho b^{4}\left(T-T_{7}\left(c_{l}\right)+c_{l} T_{1}\left(c_{l}\right)+\frac{\pi}{8}\right) \\
C_{n c F}^{1,3} & =\rho U b^{2} T_{4}\left(c_{t}\right) \\
C_{n c F}^{1,4} & =\rho U b^{2}\left(T_{4}\left(c_{t}\right)+\pi\right) \\
C_{n c F}^{2,3} & =-\rho b^{3} U\left[T_{1}\left(c_{t}\right)-T_{8}\left(c_{t}\right)-c_{t} T_{4}\left(c_{t}\right)+T_{11}\left(\frac{c_{t}}{2}\right)\right] \\
C_{n c F}^{2,4} & =-\rho b^{3} U\left[T_{1}\left(c_{l}\right)-T_{8}\left(c_{l}\right)-c_{l} T_{4}\left(c_{l}\right)+T_{11}\left(\frac{c_{l}}{2}\right)-\frac{\pi}{2}\right] \\
K_{n c F}^{1,3} & =K_{n c F}^{1,4}=0 \\
K_{n c F}^{2,3} & =-\rho b^{2} U^{2}\left(T_{4}\left(c_{t}\right)+T_{10}\left(c_{t}\right)\right) \\
K_{n c F}^{2,4} & =-\rho b^{2} U^{2}\left(T_{4}\left(c_{l}\right)+T_{10}\left(c_{l}\right)\right)
\end{aligned}
$$

The terms $T_{i j}$ are given in [40]. The size of $M_{n c F}, C_{n c F}$ is $4 \times 4$. Indeed there are two additional columns related to the flap angles $\beta_{t}, \beta_{l}$ (note that the upper left $2 \times 2$ submatrices are exactly those reported in Eq.(8)), and two additional zero rows corresponding to the equations of motion related to the flap angles. Note that the structural $M, C, K$ matrices are now enlarged to be $4 \times 4$ (and named $M_{F}, C_{F}, K_{F}$ ), to include the equations related to the flaps. Also in this case these additional rows/columns are zero, because the flaps have been assumed massless and neither damping nor stiffness are considered.

The matrices $B_{F}, C_{y F}$ (corresponding to the old $B$ and $C_{y}$ in Eq.(13)) are now given by:

$$
\begin{gathered}
\mathrm{B}_{F}^{T}=\left[\begin{array}{lllllllll}
-2 \pi \rho U b & \pi \rho U b^{2} & 0 & 0 & 0 & 0 & 0 & 0
\end{array}\right] \\
\mathrm{C}_{y F}=\left[\begin{array}{llllllll}
0 & U & T_{10}\left(c_{t}\right) \frac{U}{\pi} & T_{10}\left(c_{l}\right) \frac{U}{\pi}-U & 1 & \frac{b}{2} & T_{11}\left(c_{t}\right) \frac{b}{2 \pi} & T_{11}\left(c_{l}\right) \frac{b}{2 \pi}+b\left(c_{l}-\frac{1}{2}\right)
\end{array}\right]
\end{gathered}
$$

The aeroelastic model of the bridge including the flaps is thus cast into a generalized state space form, again similar to the case of sole deck in Eq.(12), and the corresponding $8 \times 8 E_{c}, A_{c}$ matrices are: 


$$
E_{c}=\left[\begin{array}{cc}
0 & M_{F}-M_{\mathrm{ncF}} \\
I & 0
\end{array}\right] \quad A_{c}=\left[\begin{array}{cc}
-K_{F}+K_{\mathrm{ncF}} & -C_{F}+C_{\mathrm{ncF}} \\
0 & I
\end{array}\right]
$$

The state vector is now $x=\left\{h, \alpha, \beta_{t}, \beta_{l}, \dot{h}, \dot{\alpha}, \dot{\beta}_{t}, \dot{\beta}_{l}\right\}^{T}$.

When extending this procedure to the case of FE, Eq.(16) still applies but the state vector is now augmented to $\hat{x}=\left\{Q, F, Q_{v}, F_{v}\right\}^{T}$, where $F$ and $F_{v}$ are all of the flap and angles and their derivatives respectively. Note that in this case some elements consist of just the deck (i.e. $M_{n c F}=C_{n c F}=K_{n c F}=0$ and $\left.C_{y F}=[0, U, 0,0,1, b / 2,0,0]\right)$ and some include the flaps.

\section{CONTROL Design}

We now consider the general case of a bridge having $n$ segments put in place and $k$ of them having trailing- and leading-edge flaps, see Fig.10. The uncontrolled (open loop) system is described by the aeroelastic model $P(s)$ (corresponding to the state space matrices $E_{g}, A_{g}, B_{g}, C_{z}$ ) and the leading- and trailing-edge flaps controllers are denoted as $k_{l i}(s)$ and $k_{t i}(s)$. Both controllers receive the pitch angle $\alpha_{i}$ of the corresponding element as their input, and generate the flap angles $\beta_{l i}, \beta_{t i}$ as output. The generalized state-space model is given as:

$$
\begin{aligned}
E_{g} \dot{\hat{x}} & =A_{g} \hat{x}+B_{g} u \\
z & =C_{z} \hat{x}
\end{aligned}
$$

where $u=\left\{\beta_{t 1}, \beta_{l 1}, \ldots, \beta_{t k}, \beta_{l k}\right\}^{T}$ and $z=\left\{\alpha_{1}, \ldots \alpha_{k}\right\}^{T}$. Since the controlled inputs are the flap angles rather than torques to massy flap bodies, the model given in Eq.(46),(47) must generate the first and second derivatives of the flap angles internally; these derivatives are required by the aerodynamic model. This is achieved using a generalized state space system of the form:

$$
\left[\begin{array}{ll}
0 & 0 \\
1 & 0
\end{array}\right]\left[\begin{array}{l}
\dot{x}_{1} \\
\dot{x}_{2}
\end{array}\right]=\left[\begin{array}{ll}
1 & 0 \\
0 & 1
\end{array}\right]\left[\begin{array}{l}
x_{1} \\
x_{2}
\end{array}\right]+\left[\begin{array}{c}
-1 \\
0
\end{array}\right] \beta
$$

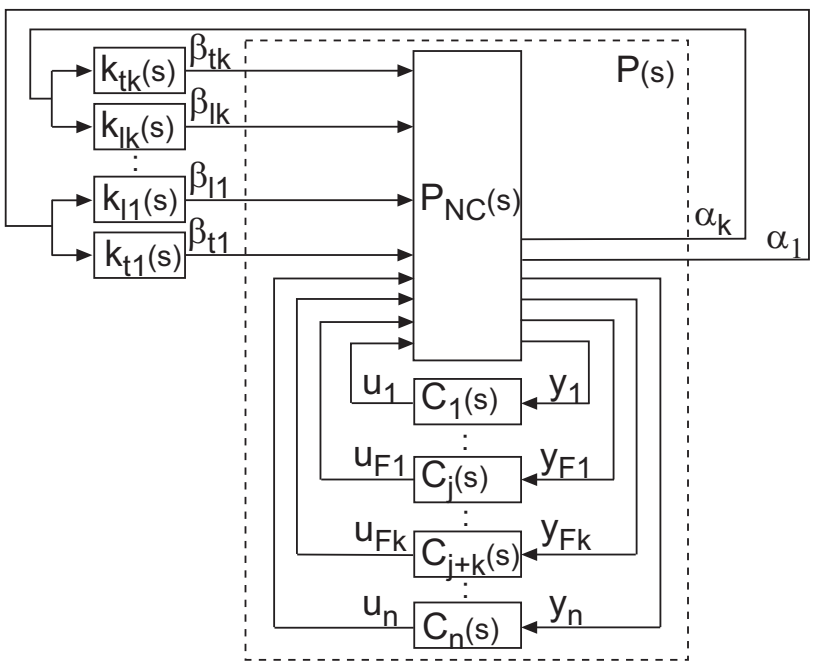

Fig. 10: Block diagram of the system: $P_{N C}(s)$ is the aeroelastic model without the circulatory terms, $P(s)$ is the aeroelastic model (the $C_{i}(s)$ feedbacks are for the inclusion of the circulatory terms), $k_{l i}(s)$ and $k_{t i}(s)$ are the compensators of the leading and trailing flaps, $\alpha_{i}$ are the pitch angles, $\beta_{l i}$ and $\beta_{t i}$ are the leading- and trailing-edge flap angles. 
The normalized left coprime factorization of the plant $P$ is defined as:

$$
P=M^{-1} N
$$

where $M, N \in H_{\infty}$ and

$$
M M^{\sim}+N N^{\sim}=I
$$

$(\cdot)^{\sim}$ means adjoint. The perturbed plant is:

$$
P_{\Delta}=\left(M-\Delta_{M}\right)^{-1}\left(N+\Delta_{N}\right)
$$

The aim is to design a stabilizing controller that maximizes the size of admissible perturbation:

$$
\left\|\Delta_{M} \quad \Delta_{N}\right\|_{\infty}<\gamma^{-1}
$$

thereby minimizing the achievable value of $\gamma[14,35]$ :

$$
\left\|\left[\begin{array}{c}
K \\
I
\end{array}\right](I-P K)^{-1}\left[\begin{array}{ll}
P & I
\end{array}\right]\right\|_{\infty}<\gamma=\frac{1}{\varepsilon}
$$

In practice the objective is to find a stabilizing feedback controller that minimize $\gamma$ while satisfying Eq.(54) ( $\varepsilon$ is called normalized coprime stability margin or gap metric stability margin). Note that it is always $\gamma \geq 1$. It is worth noting that minimizing $\gamma$ results in a bounded $\infty$-norm for $K S, S, K S P, S P$ (this terms are the four sub-matrices obtained when expanding the left hand side of Eq.(54)), where $S=(1-P K)^{-1}$. These terms are robust stability indexes: the lower their norm, the better the related robustness. In particular $\|K S\|_{\infty}$ is related to the robustness against additive perturbation on the plant $P,\|S\|_{\infty}$ is related to the robustness against additive perturbation on the loop transfer function $P K$, 


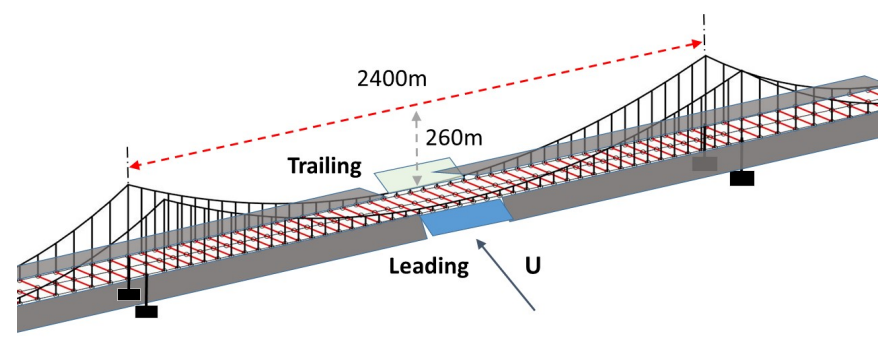

Fig. 11: Bridge with flaps considered to be an integral part of the deck.

$\|K S P\|_{\infty}$ is related to the robustness against multiplicative perturbation on the controller $K$ and $\|S P\|_{\infty}$ is related to the robustness against additive perturbation on controller $K$.

We use the $H_{\infty}$ control theory framework to solve this robust stabilization problem. This will result in the optimal controller, i.e. the (active) controller that gives the maximum/best stability robustness and will serve as a reference for simpler controllers, e.g. lower order and/or passive controllers. A characteristics of the $H_{\infty}$ optimal controller is that it has size equal to that of the plant reduced by at least one. Many times controllers that are slightly sub-optimal (i.e. controllers giving $\gamma>\gamma_{\min }$ ) are derived for a number of reasons (numerically easier to derive, smaller gains, etc.). These controllers have the same degree of the plant. It is now clear that in general these optimal and suboptimal controllers are complex and have a very high degree. However model reduction techniques on model and/or controller can be implemented for practical implementation $[14,35]$.

\section{Design EXAMPle}

Since the critical flutter and divergence speed of the Great Belt Bridge are already very high a longer version of the Bridge was created. All the parameters in Tab.I are kept the same but for this case the main span has a length of $L_{m}=2400 \mathrm{~m}$ (instead of $1620 \mathrm{~m}$ ), the cable sag is $f=260 \mathrm{~m}$ (instead of $180 \mathrm{~m}$ ) and the length of the shortest hanger remains $h_{e, \min }=10 \mathrm{~m}$. In practice the main span and the towers have been upscaled by $50 \%$, while the same deck section is kept the same. The controllable winglets are concentrated in the middle of the main span so as to suppress the first critical flutter mode and are considered to be an integral part of the deck as shown in Fig.11.

For this bridge example the flutter and divergence speed using thin aerofoil theory is estimate at $57 \mathrm{~m} / \mathrm{s}$ and $68 \mathrm{~m} / \mathrm{s}$ respectively. As shown in the left hand part of Fig. 12 there is a second flutter mode becoming unstable at $73 \mathrm{~m} / \mathrm{s}$. Each point on the root-locus diagram corresponds to a mode of the system at a specific wind speed. The right hand part of Fig. 12 presents the natural frequency and the damping ratio of different modes along the first instability path. Negative aerodynamic damping signifies the onset of an aeroelastic instability and the corresponding wind speed is indicated on the plot. At zero wind speed the natural frequency and damping ratio is that of the first torsional mode while the following points do not correspond to a pure structural mode since the airflow alters the system.

For points along instability paths the corresponding complex eigenvectors are extracted to give insight in the mode coupling which result in aerodynamic instability. Fig. 13 presents the normalized vertical projection of the complex vertical and torsional real component of the mode for the two flutter instabilities. The left part of Fig.13 (first flutter mode) reveals the contribution of higher vertical modes 


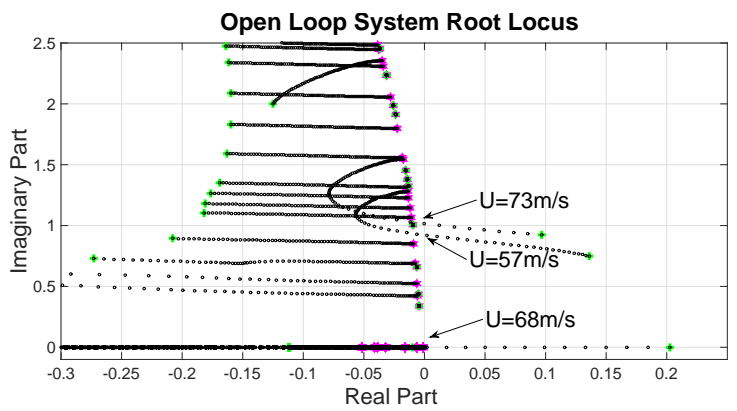

(a)
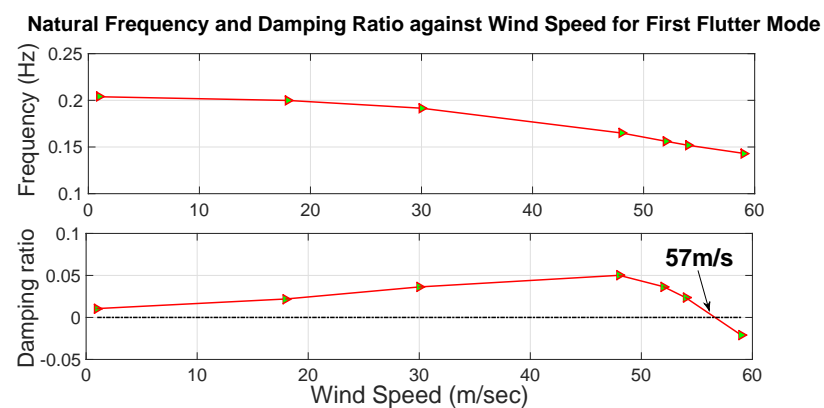

(b)

Fig. 12: (a) Root locus of the $2400 m$ main span bridge example. The first flutter mode becomes unstable at $57 \mathrm{~m} / \mathrm{s}$ and the second one at $73 \mathrm{~m} / \mathrm{s}$. The torsional divergence mode reaches instability at $68 \mathrm{~m} / \mathrm{s}$. (b) Natural frequency $(\mathrm{Hz})$ and damping ratio for differen wind speeds along the first flutter mode. The system becomes unstable when damping is negative.

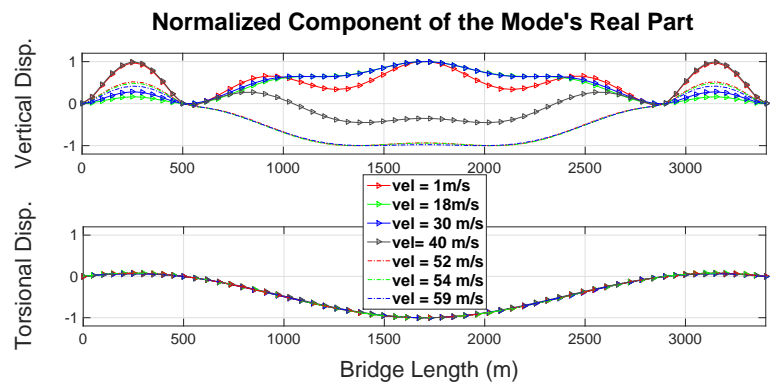

(a)

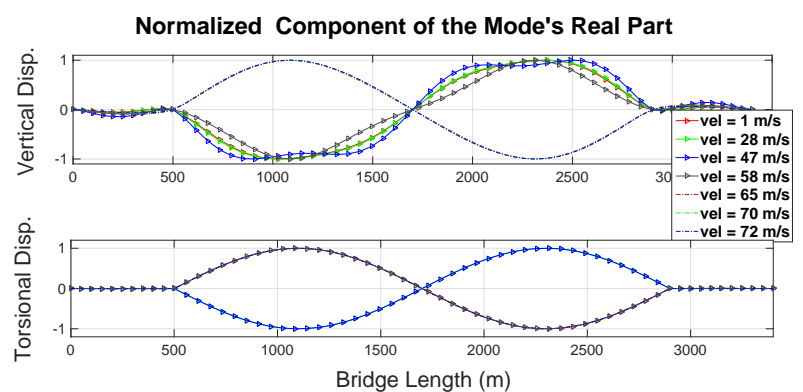

(b)

Fig. 13: Mode shape of the first flutter mode (a) and the second flutter mode (b).

near flutter speed.

The goal in this design exercise is to determine the minimum flap area needed stretching from the midpoint of the main span to restore the stability limits to that of the Great Belt Bridge. The system's robust stability margins up to wind speed of $80 \mathrm{~m} / \mathrm{s}$ are examined while varying the length of the flaps. As a first step we follow a simplified procedure for the controller design as we optimize for coprime robustness on the equivalent sectional model, Fig.15. Fig.14(a) presents the robustness index $\gamma$ of the sectional model as a function of wind speed for the case of the open loop system (no control) as well as for different control configurations. In this case the trailing-and leading edge flaps have a width of $3 m$ each. The blue crosses correspond to the optimal $H_{\infty}$ controller, optimized for coprime robustness at every wind speed (adaptive). These controllers are of $9^{\text {th }}$ order which is slightly smaller than that of the plant which is $12^{\text {th }}$ order. Green hexagons indicate the robustness index for suboptimal controllers, designed to have a $\gamma$ which is $10 \%$ larger than the optimal $\gamma$. Again they are designed for every wind speed.

For the sake of comparison we superimpose the robustness index of a reduced stabilizing controller, which comprises of a first order leading and a second order trailing flap compensator. Note that, although this controller is much leftward in the complex plane, its robustness is significantly lower, indicating that the distance from the imaginary axis is not a good indicator for robustness at it has been suggested in previous studies. Fig.14a demonstrates that, as expected, the robustness index approaches infinity, zero robustness, at flutter instability, $57 \mathrm{~m} / \mathrm{s}$ for the open loop system. The performance of the optimal and suboptimal controllers is very similar and both control approaches achieve good margins 
up to $67-68 \mathrm{~m} / \mathrm{s}$, before the torsional divergence speed. However system stabilization above torsional divergence has a significant toll on robustness performance. The corresponding root-loci indicate that all closed loop systems are stable up to $80 \mathrm{~m} / \mathrm{s}$ however achieving good robustness margins is a much more challenging objective.

The optimal controller configuration derived on the section model is then applied to the three dimensional bridge model. In this case, The controller matrix has diagonal structure whereas the same control law is applied to the flaps of each element with each element. The system output is the pitch of the element deck and the input is the rotation of the flap angles. Fig.16(a) presents the robustness index of the entire structure with flaps along the full length, half-length and quarter-length of the $2400 \mathrm{~m}$ main span. It is easily observed that the controller designed on the section model has poor robustness margin when applied to the full bridge. It is evident that the system loses robustness rapidly for the case when $25 \%$ of the span is equipped with winglets while for longer flap surfaces an abnormal spike is observed indicating loss of robustness for certain wind speeds.

Fig.16(b) are similar graphs with the difference that the suboptimal controller designed from the sectional model is now applied to the FE model. These figures indicate this strategy yields much smoother robustness margins, without significant loss in performance. In addition, suboptimal controller design is less computationally demanding. Flaps along 50\% of the span's length have closely comparable performance to the case of flaps along the $100 \%$ of the length. When the wind speed approaches the second flutter instability, $73 \mathrm{~m} / \mathrm{s}$, as expected these controllers are ineffective (robustness index becomes infinite), since the sectional model does not consider those modes.

The final investigation consists in applying the controller design procedure directly on the FE model, for different flap lengths located around the midpoint. Fig.16(c) presents the coprime robustness index for those cases. In this modelling example the main span is modelled using 37 elements (about $65 \mathrm{~m}$ width each). It is worth stressing that Fig.16(c) summarizes the best achievable results for enhancing the aeroelastic performance for the chosen model, and quantifies the effectiveness of using trailing/leading-edge flaps while retaining good coprime robustness margins. The configuration with 11 flaps, approximately $30 \%$ of the main span length, appears to be a reasonable choice as the aerodynamic instability limit is increased up to $70 \mathrm{~m} / \mathrm{s}$ with a robustness index $\gamma \approx 5$. Achieving good robustness above the open loop divergence speed and the second flutter mode is very difficult, with a reasonable flap width, even by covering the entire span length with controllable surfaces.

The controller design procedure based on the FE model results in a very high order controller. For implementation purposes it is important to implement a reduced order approximation, preferably with

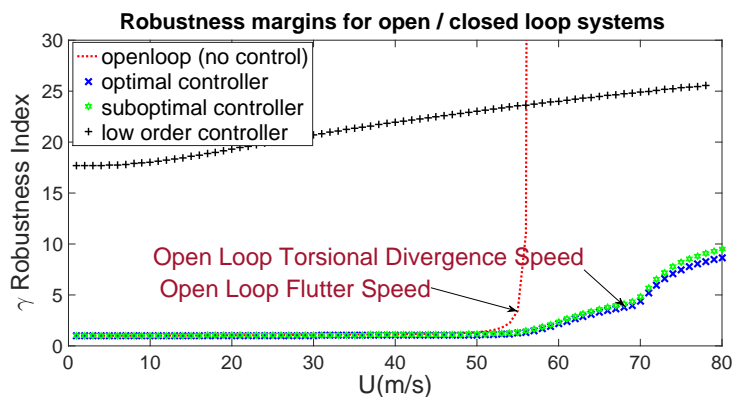

(a)

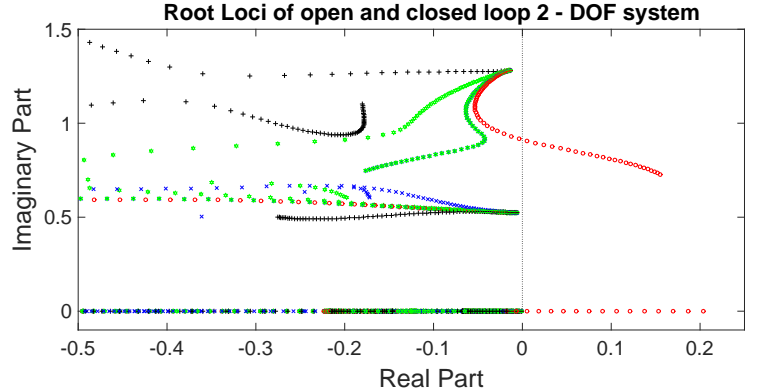

(b)

Fig. 14: (a) Robustness index as a function of wind speed for different control configurations. (b) Corresponding root loci diagrams for the different controllers. 


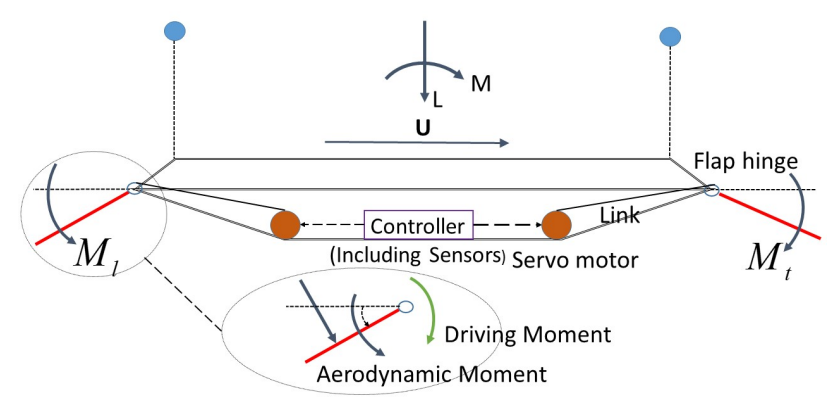

Fig. 15: Sketch of the practical implementation of the active control system to the bridge deck. The pitch of the deck is monitored and the control signals are transmitted to the servo motors which determine the rotation angles of the leading and trailing flaps.

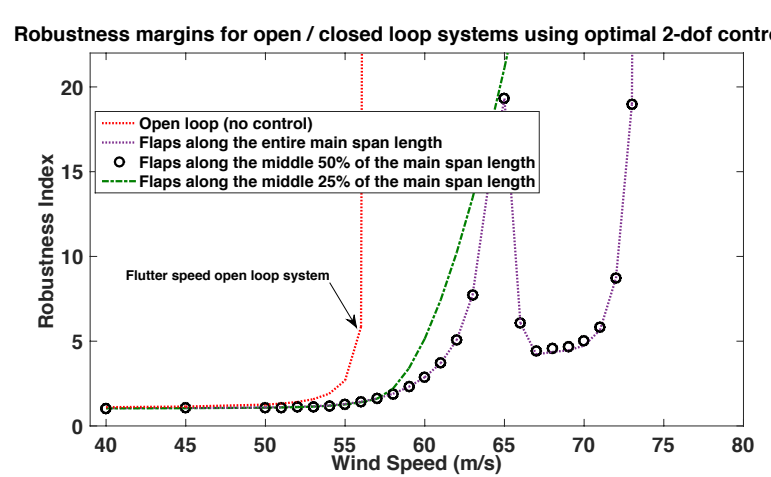

(a)

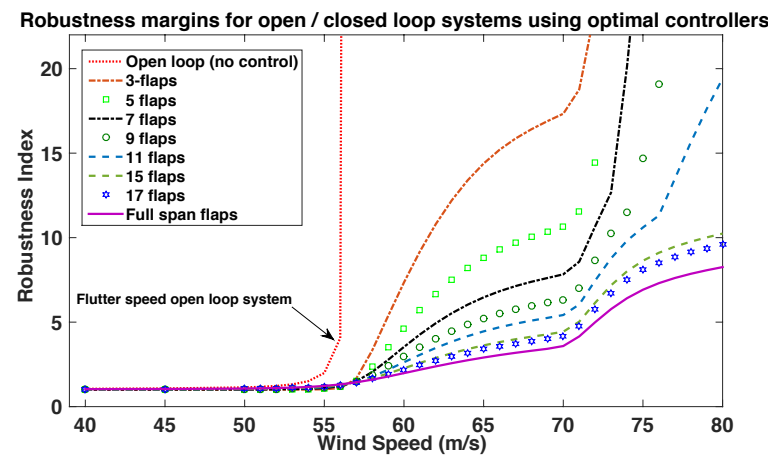

(c)

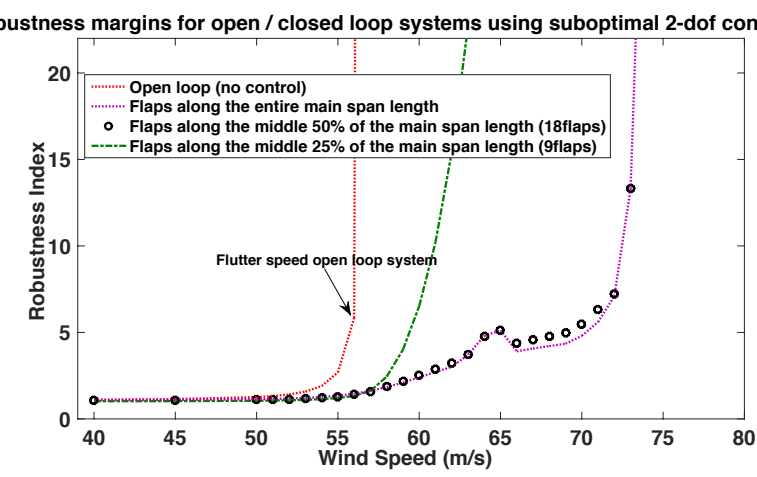

(b)

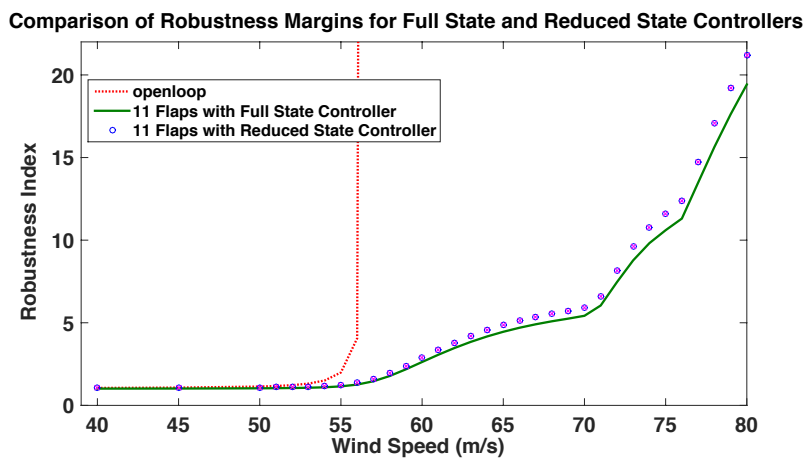

(d)

Fig. 16: Coprime robustness index of the full FE model as a function of wind speed. (a) Optimal controller designed on the sectional model. (b) 10\%-suboptimal controller designed on the sectional model. (c) Optimal controller designed on the full FE model. (d) Reduced order optimal controller designed on the full FE model. 

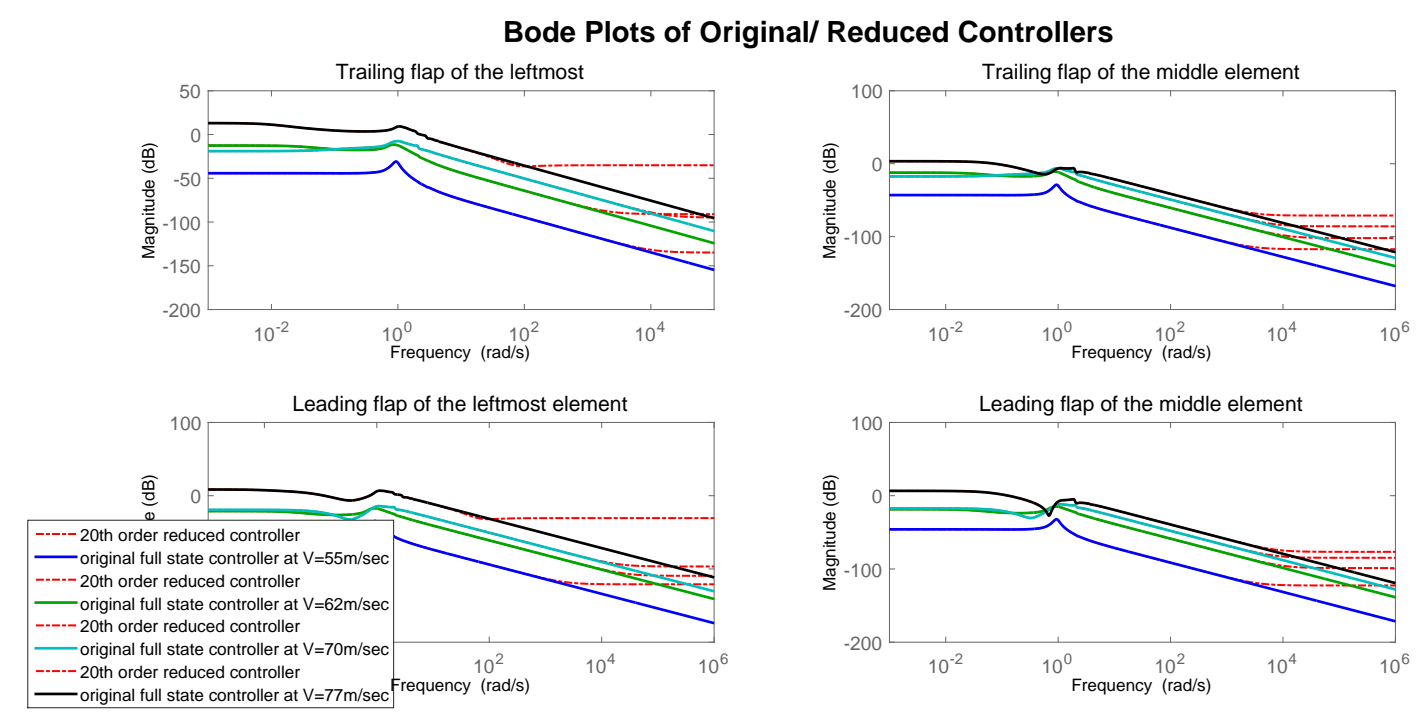

Fig. 17: Transfer functions related to four of the $22 \times 11$ entries of the control matrix: leftmost leadingand trailing-edge flap motion from the pitch of the leftmost element, and middle leading- and trailingedge flap motion from the pitch of the middle element.

little sacrifice in the performance. There exist several reduction procedures and for the task at hand $[14,35]$ we perform a truncation operation to the balanced realization of the state space representation of the optimal controller matrix. With this procedure we are able to reduce substantially (from $400+$ to 20 states) the size of the controllers with a negligible loss in performance: Fig.16(d) compares the robustness of the system with full state and reduced order controller respectively.

In the frame of this design exercise time simulations at different wind speeds are performed for the case of approximatively $30 \%$ of the main span controlled (11 leading and 11 trailing edge flaps), consequently the controller matrix has dimensions 11 by 22. In Fig.17 four of the 242 entries of the matrix are presented; namely the ones related to the leading- and trailing-edge flap motion from the pitch of the leftmost element, and the those related to the leading- and trailing-edge flap motion from the pitch of the middle element. Different wind speeds are considered for presentation namely: $55 \mathrm{~m} / \mathrm{s}$ (below the first open loop critical flutter speed), $62 \mathrm{~m} / \mathrm{s}$ (above the first critical flutter speed and below the torsional divergence speed), $70 \mathrm{~m} / \mathrm{s}$ (above the torsional divergence and below the second flutter speed) and $77 \mathrm{~m} / \mathrm{s}$ (above the second flutter speed). The $20^{\text {th }}$ state, reduced order, controller is superimposed to the full size controller, showing once again that the two are almost identical in the spectrum of frequencies of interest.

Fig.18 presents the maximum magnitudes of the controller matrix for the two highest wind speeds considered: $70 \mathrm{~m} / \mathrm{s}$ and $77 \mathrm{~m} / \mathrm{s}$. It is interesting to note that at $70 \mathrm{~m} / \mathrm{s}$ the flaps in the middle have slightly higher gains than the flap in the edges and that trailing flaps move more than the leading edge flaps. On the contrary at $77 \mathrm{~m} / \mathrm{s}$ there is an asymmetric distribution of magnitudes, due to the flap controller's effort to suppress the first asymmetric torsional mode.

Fig.19 presents the control effectiveness in time domain for suppressing pitching and heaving of the bridge at wind speeds $62 \mathrm{~m} / \mathrm{s}$ and $77 \mathrm{~m} / \mathrm{s}$. The initial condition chosen is that of a sinusoidal twisting/pitch, of the main deck with its maximum value at the midpoint of $2 \mathrm{deg}=0.035 \mathrm{rad}$. The transient response of the deck's heave and pitch is presented for two different points (middle and one third span) for the open-loop system (no flaps active), and the closed-loop system with the 11 flaps around the midpoint (30\% of the whole span). When the wind speed is of $62 \mathrm{~m} / \mathrm{s}$ the open loop 
Maximum gains of controller matrix entries at wind speed $70 \mathrm{~m} / \mathrm{sec}$

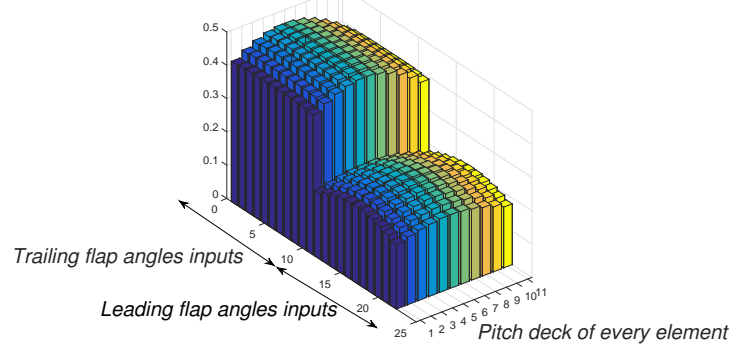

(a)

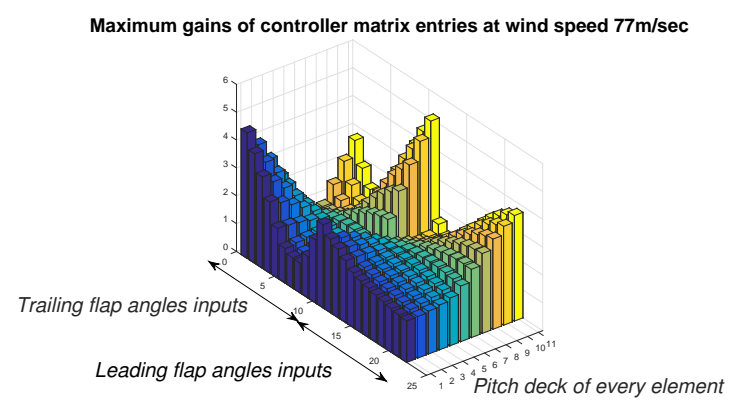

(b)

Fig. 18: Maximum magnitudes of the $22 \times 11$ transfer functions of the reduced order controller matrix for wind speeds of $70 \mathrm{~m} / \mathrm{s}$ (a) and $77 \mathrm{~m} / \mathrm{s}$ (b)
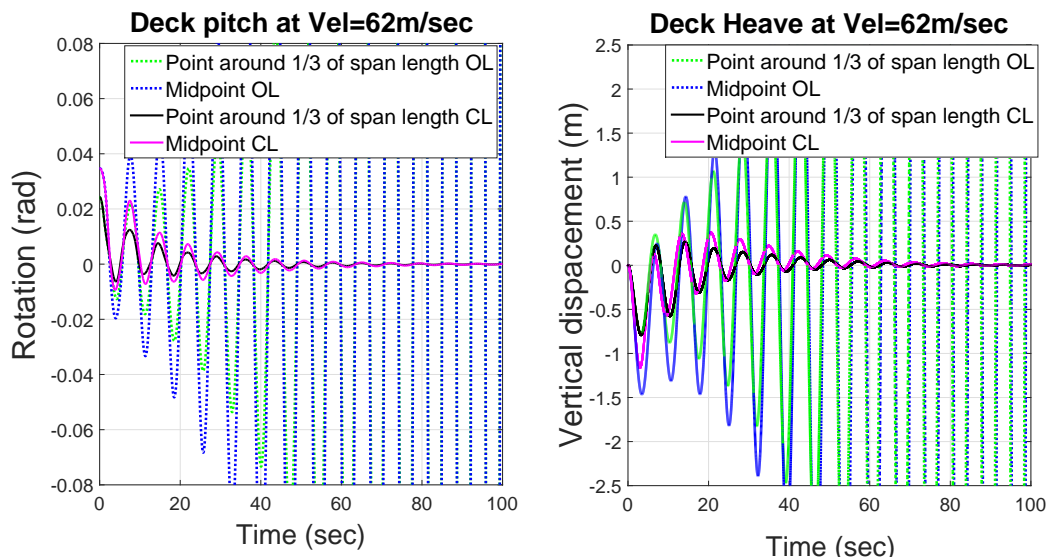

(a)
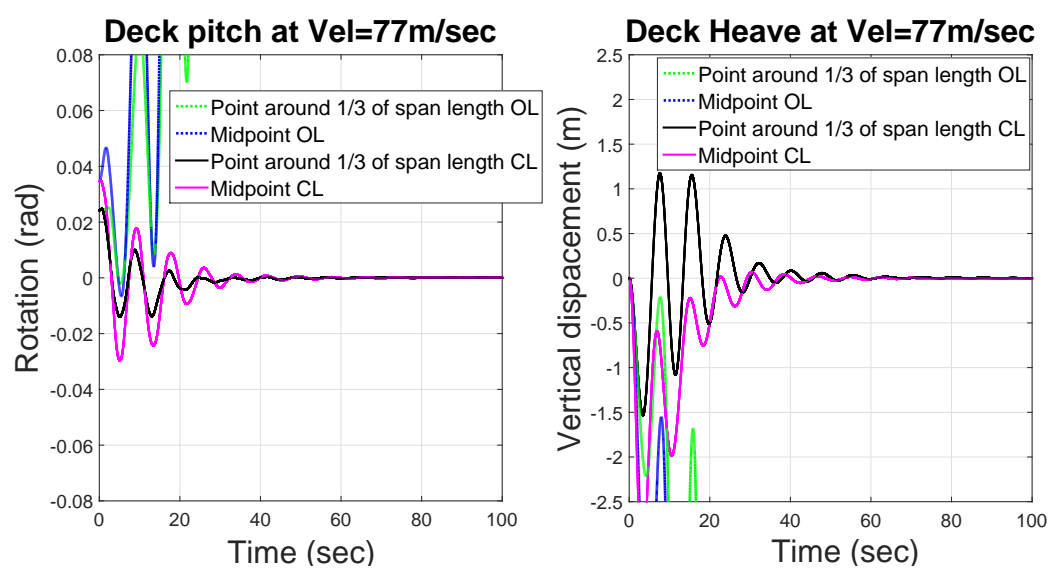

(b)

Fig. 19: Deck's Pitch and Heave transient response at wind speed of $62 \mathrm{~m} / \mathrm{s}$ (a) and $77 \mathrm{~m} / \mathrm{s}$ (b) for the open-loop (no controller) and closed-loop system, case 11 flaps around the middle (30\% of the whole span), reduced order controller. 
system diverges in an oscillatory motion due to flutter instability whereas for a wind speed of $77 \mathrm{~m} / \mathrm{s}$ the instability is much more violent as this is a combination of flutter and torsional divergence. It is worth noting that this fictitious simulation is the worst case example as if we switch on the flap controllers at the chosen wind speed under the given initial condition. In reality the controllers would already be functioning at lower wind speeds thus preventing the deck pitch from reaching large values.

The movement of the flaps with regard to the deck pitch is shown in Fig.20, again for the same wind speeds and initial conditions. Rotations of the leading and trailing flaps at three different locations are presented (middle, leftmost, midway). The time history plots give insight into the flap behaviour for suppressing deck motion. At $62 \mathrm{~m} / \mathrm{s}$ (and in general for all wind speeds lower than the second flutter speed) there is a similar behaviour in the movement of the trailing and leading flaps at different locations, showing that potentially the same control law can be applied for all flaps along the span. On the other hand this is not the case at $77 \mathrm{~m} / \mathrm{s}$. In addition, note that the flap rotations are restricted to less than $10 \mathrm{deg}$ in the simulation reported. Of course this depends on the initial condition. However the transfer function magnitudes show that for wind speeds up to $80 \mathrm{~m} / \mathrm{s}$ the rotation of the flaps does not exceed $15 \mathrm{deg}$ given a deck pitch of $2 \mathrm{deg}$. This is an important observation as larger flap rotations cause flow separations and thin aerofoil theory is no more valid. At this point it is reminded that our analysis is based on thin airfoil theory and larger gains for the trailing flap might be required due to flow separation caused by deck bluffness. In [12] it was indeed concluded that a 2 to 3 times larger flap deflection is needed than the original controller computation. This finding however, did not pose a serious issue as the flap angle never exceeded $6^{\circ}$ in the experiments.

\section{CONCLUSION}

The work addressed the issue of suppressing aerodynamic instabilities such as flutter and torsional divergence on long-span suspension bridges using trailing- and leading-edge controllable flaps in combination. The initial part of this study dealt with the mathematical formulation for capturing the essential aeroelastic characteristics of suspension bridges using a reduced size structural model. Classical results from potential flow theory were recast in a form suitable for control analysis and design, implementing therein the transformation of the wing-aileron-tab to the flap-deck-flap configuration, using feedback as well as rational approximation of the circulatory terms, which differentiates this work from previous analyses. The results were compared to previous experimental and analytic studies finding good agreement. The aeroelastic finite element procedure was then extended to implement a rational approximation of the experimentally obtained flutter derivatives. The analysis showed that, for the Great Belt Bridge, the system's aerodynamic behaviour is similar to that predicted by thin aerofoil theory, thus supporting the use of the latter for all subsequent investigations using control surfaces.

In the control part of this work $H_{\infty}$ control theory was employed to tackle the robust stabilization of the unstable bridge at high wind velocities. This novel approach successfully quantifies structural and aerodynamic uncertainty and answers questions related to the applicability of a control strategy based on controllable winglets. It was shown that, although the stabilization of the system can be obtained relatively easily, the closed loop system loses robustness rapidly for wind speeds beyond the open loop torsional divergence speed. This is an important result that sets a qualitative limit for the performance of all controllers using leading- and trailing-edge winglets. However, by fitting the deck with controllable flaps of about $30 \%$ of the main span's total length, it was possible to significantly increase the structure's aeroelastic limits with good robustness margins, i.e. the flutter critical speed was raised up to the torsional divergence critical speed. This work demonstrates the feasibility of the proposed aeroelastic control methodology and although further risk analysis and experimental investigation is needed the results are encouraging for real bridge applications. The presented high order active controller implementation also serves as a benchmark for ongoing work on using low order passive flap controllers, which avoid the use of an external power source and sensors. 

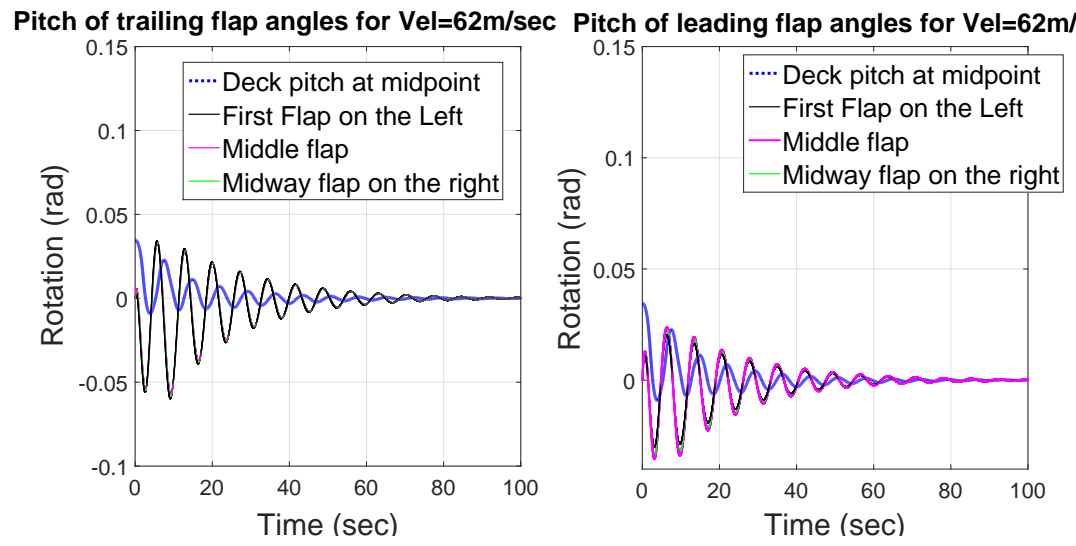

(a)
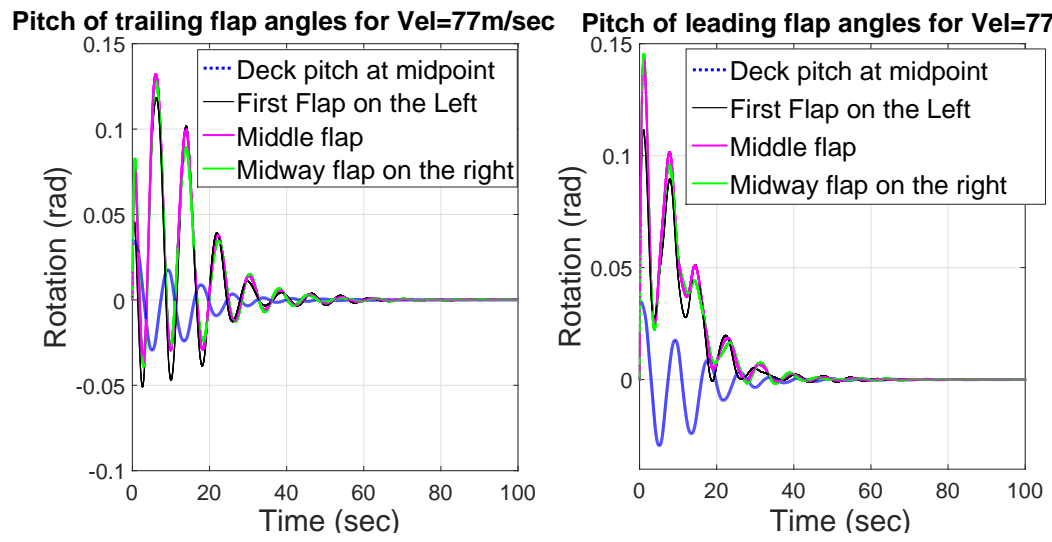

(b)

Fig. 20: Flap angles transient response at wind speed of $62 \mathrm{~m} / \mathrm{s}$ (a) and $77 \mathrm{~m} / \mathrm{s}$ (b) at three different locations, case 11 flaps around the middle (30\% of the whole span), reduced order controller.

\section{ACKNOWLEDGEMENTS}

This work was partially funded by the IKY-State Scholarship Foundation by means of the ESPA European fund 2007-2013 and supported by a Marie Curie Intra European Fellowship within the 7th European Community Framework Programme.

\section{REFERENCES}

[1] A.M. Abdel-Ghaffar. Free lateral vibrations of suspension bridges. Journal of Structural Division ASCE, 104(ST3):503-525, 1978.

[2] A.M. Abdel-Ghaffar. Free torsional vibrations of suspension bridges. Journal of Structural Division ASCE, 105(ST4):767-788, 1979.

[3] A.M. Abdel-Ghaffar. Free vertical vibrations of suspension bridges. Journal of Structural Division ASCE, 105(ST10):2053-2076, 1980.

[4] M. A. Astiz. Flutter stability of very long span bridges. Journal of Bridge Engineering, 3(3):132-139, 1998.

[5] K. N. Bakis, M. Massaro, M. S. Williams, and D. J. N Limebeer. Aeroelastic control of long span suspension bridges during erection. In IABSE conference, Elegance in Structures, Nara,Japan, 2015.

[6] B. Basu, O. S. Bursi, F. Casciati, S. Casciati, A. E. D. Grosso, M. Domaneschi, L. Faravelli, J. H. Szulc, H. Irschik, M. Krommer, M. Lepidi, A. Martelli, B. Ozturk, F. Pozo, G. Pujol, Z. Rakicevic, and J. Rodellar. A european association for the control of structures joint perspective. recent studies in civil structural control across europe. Journal of Structural Control and Health Monitoring, 21:1414-1436, 2014.

[7] R. L. Bisplinghoff, H. Ashley, and R. L. Halfman. Aeroelasticity. Dover Publications, New York, 1996.

[8] C. M. Casado, I. M. Diaz, J. Sebastian, A. V. Poncela, and A. Lorenzana. Implemenation of passive and active vibration control on an in-service foorbridge. Journal of Structural Control and Health Monitoring, 20:70-87, 2013. 
[9] X. Chen, M. Matsumoto, and A. Kareem. Aerodynamic coupling effects of flutter and buffeting of bridges. Journal of Engineering Mechanics, 126:17-26, 2000

[10] D. Cobo. An analysis of wind stability. Improvements to the response of suspension bridges. Tesis doctoral de la Universitat de Catalunya, 1998.

[11] G. Diana, C. Federico, Z. Alberto, A. Colina, and S. Bruni. Aerodynamic design of very long-span suspension bridges. In Long-Span and High-Rise Structures, IABSE Symposium, pages 115-130, Kobe, 1998.

[12] K. Gouder, X. Zhao, D. J. N Limebeer, and J. M. R Graham. Experimental aerodynamic control of a long-span suspension bridge section using leading and trailing edge control surfaces. IEEE Transactions on Control System Technology, xx:xx, submitted.

[13] J. M. R. Graham, D. J. N. Limebeer, and X. Zhao. Aeroelastic control of long-span suspension bridges. Journal of Applied Mechanics, 78:041018-1 to 041018-12, 2011

[14] M. Green and D. J. N. Limebeer. Linear Robust Control. Prentice Hall, Englewood Cliffs, New Jersey, 1995.

[15] H. I. Hansen and P. Thoft-Christensen. Active flap control of long suspension bridges. Journal of Structural Control, 8(1):33-82, 2001.

[16] H. M Irvine. Studies in the Statics and Dynamics of Simple Cable Systems. California Institute of Technology, Technical Report, 1974.

[17] A. Jain, N.P. Jones, and R.H. Scanlan. Coupled flutter and buffeting analysis of long-span bridges. Journal of Structural Engineering, ASCE, 122:716-725, 1996.

[18] R.T. Jones. Operational treatment of the nonuniform-lift theory. NACA Report, TR-667, 1938.

[19] J. A. Jurado, S. Hermandez, F. Nieto, and A. Mosquera. Bridge Aeroelasticity: Sensitivity Analysis and Optimum Design. WITpress, 2011.

[20] S. D. Kwon, M. S. S. Jung, and S. P. Chang. A new passive aerodynamic control method for bridge flutter. Journal of Wind Engineering and Industrial Aerodynamics, 86:187-2002, 2000.

[21] A. Larsen. Aerodynamic aspects of the final design of the $1624 \mathrm{~m}$ suspension bridge accross the greal belt. Journal of Wind Engineering and Industrial Aerodynamics, 48:261-285, 1993.

[22] K. Li, Y. J. Ge, Z. W. Guo, and L. Zhao. Theoretical framework of feedback aerodynamic control of flutter oscillation for long-span suspension bridges by the twin-winglet system. Journal of Wind Engineering and Industrial Aerodynamics, 145:166-177, 2015.

[23] D. J. N. Limebeer, J. M. R. Graham, and X. Zhao. Buffet suppression in long-span suspension bridges. Annual Reviews in Control, 35:235-246, 2011.

[24] G. B. R. Lopes and R. S. Silva. Time domain modelling and bridge deck flutter and applications. Mecanica Computational, 14:529-5553, 2010.

[25] K. Makoto. Technology of the akashi kaikyo bridge. Journal of Structural Control and Health Monitoring, 11:75-90, 2004.

[26] M. Matsumoto, K. Mizuno, K. Okubo, Y. Ito, and M. Matsumiya. Flutter instability and recent development in stabilization of structures. Journal of Wind Engineering and Industrial Aerodynamics, 95:888-907, 2007.

[27] Albrecht P. Namini, A.H. and H. Bosch. Finite element based flutter analysis of cable suspended bridges. Journal of Structural Engineering ASCE, 118(6):1509-1526, 1992.

[28] P. Omenzetter, K. Wilde, and Y. Fujino. Study of passive deck-flaps flutter control system on full bridge model 1: Theory. Journal of Engineering Mechanics, March:264-279, 2002.

[29] P. Omenzetter, K. Wilde, and Y. Fujino. Study of passive deck-flaps flutter control system on full bridge model 2: Results. Journal of Engineering Mechanics, March:280-286, 2002.

[30] K. H. Ostenfeld and A. Larsen. Bridge engineering and aerodynamics. In In Aerodynamics of Large Bridges, Proceedings of the First International Symposium on Aerodynamics of Large Bridges, Copenhagen,Denmark, 1992.

[31] K. L. Roger. Airplane math modelling methods for active control design. AGAPO-CP-228, New York, 1997.

[32] R. H. Scanlan. Problematics in formulation of wind-force model for bridge deck. Journal of Engineering Mechanics, 119:13531375, 1993.

[33] R. H. Scanlan. Observations on low-speed aeroelasticity. Journal of Engineering Mechanics, 128:1254-1258, 2012.

[34] R. H. Scanlan and J. J. Tomko. Airfoil and bridge deck flutter derivatives. Journal of Engineering Mechanics, 97(6):1717-1737, 1971.

[35] S. Skogestad and I. Postlethwaite. Multivariable Feedback Control: Analysis and Design. 2nd Ed. Wiley, 2006.

[36] U. Starossek. Prediction of bridge flutter through use of finite elements. Structural Engineering review, 5(4):301-307, 1993.

[37] U. Starossek and H. Aslan. Passive control of bridge deck flutter using tuned mass dampers and control surfaces. In 7th European Conference on Strcutural Dynamics, Southampton,United Kingdom, 2008.

[38] U. Starossek, H. Aslan, and L. Thiesemann. Experimental and numerical identification of flutter derivatives for nine bridge deck sections. Wind and Structures, 12 No.6:519-540, 2009.

[39] T. Theodorsen. General theory of aerodynamic instability and the mechanisms of flutter. NACA Report, TR-496, 1934.

[40] T. Theodorsen and I. E. Garrick. Nonstationary flow about a wing-aileron-tab combination including aerodynamic balance. $N A C A$ Report, TR-736, 1942.

[41] L. Tuanjie and M. Yujuan. Robust vibration control of flexible tensegrity structure via $\mu$ synthesis. Journal of Structural Control and Health Monitoring, 20:173-186, 2011.

[42] K. Wilde and Y. Fujino. Variable-gain control applied to aerodynamic control of bridge deck flutter. pages 682-687. Proceedings of 35th Conference on Decision and Control, Kobe, Japan, 1996.

[43] K. Wilde and Y. Fujino. Aerodynamic control of bridge deck flutter by active surfaces. Journal of Engineering Mechanics, 124(7):718-727, 1998. 